Heiko Hausendorf

\title{
Interaktion im Raum \\ Interaktionstheoretische Bemerkungen zu einem vernachlässigten Aspekt von Anwesenheit
}

\begin{abstract}
Es ist nicht zu übersehen, dass die neuere Gesprächsforschung der Zeitlichkeit der Interaktion, also der Organisation von Nacheinander und Reihenfolge, viel mehr Aufmerksamkeit geschenkt hat als der Räumlichkeit der Interaktion, also der Organisation der Verortung und Platzierung Anwesender im Raum. Für Zeitlichkeit haben wir mit dem Konzept der Sequentialität eine methodologische Leitorientierung, die sich in unzähligen Analysen empirisch bewährt hat und theoretisch explizierbar ist. Für Räumlichkeit gilt das (trotz der Pionierarbeiten von Goffman, Hall, Birdwhistell, Kendon und anderen) offenkundig nicht, obwohl man mit Fug und Recht behaupten kann, dass Raum und Räumlichkeit - genauso wie Zeit und Zeitlichkeit - interaktiv in Anspruch genommen und interaktiv hergestellt werden. Kann man daraus schließen, dass der Raum für die Interaktion weniger wichtig ist als die Zeit? Oder hat es damit zu tun, dass Raum und Räumlichkeit für eine an sprachlichen Erscheinungsformen orientierte linguistische Gesprächsforschung empirisch und methodisch schwerer zu fassen sind? Und welcher Art sind die Phänomene, die man zu sehen bekommt, und die Konzepte, die man braucht, wenn man Räumlichkeit als Aspekt von Anwesenheit zu thematisieren versucht? Was schließlich leistet Sprache für die interaktive Inanspruchnahme von Raum und Räumlichkeit?

Diesen und ähnlichen Fragen geht der folgende Beitrag am Beispiel einer Interaktionsepisode in einer Kunstausstellung nach.
\end{abstract}

\section{Einleitung}

Wenn wir sprechen und zuhören, setzen wir einen umgebenden Raum fast immer voraus. In vielen Fällen ist das ein architektonisch irgendwie gestalteter, „umbauter" oder ,freier“, öffentlicher oder privater Raum; jedenfalls ein Raum, in dem sich die Interaktion abspielt. Es ist das „hier“, von dem keine Interaktion und kein Gespräch abstrahieren können. Sprechen und Zuhören geschieht nie raum- und ortlos, wie sehr dieses „hier“ in einer konkreten Interaktionsepisode oder im Verlauf der Interaktion auch immer in den Hintergrund treten mag. Es sind die weniger sprachlastigen Interaktionsereignisse, die uns diese Raum- und Ortsbindung der Interaktion sofort deutlich vor Augen führen: Man denke an einen Boxkampf oder ein Fußballspiel, wenn man Beispiele sucht für Interaktionen, in denen zwar auch gesprochen und zugehört wird, in denen aber primär raumbezogen inter- 
agiert wird. Nicht zufällig hat man vom Fußballer (nicht vom Fernsehkommentator) Günther Netzer gesagt, dass er aus der Tiefe des Raumes kam, wenn man seine Interaktionsfähigkeiten auf dem Spielfeld beschreiben wollte.

Wer so argumentiert, argumentiert von der Interaktion her - und es ist kein Zufall, dass Erving Goffman, einer der Pioniere dieser Denkrichtung, von Anfang an neben das Gespräch den Boxkampf (und das Kartenspiel, das Tanzpaar oder das Chirurgieteam im Operationssaal) als gleichrangige Beispiel für fokussierte Interaktion gestellt hat (Goffman 1964, S. 135). Argumentiert man dagegen stärker von sprech- und zuhörlastigen Interaktionen wie dem „Gespräch“ her, ist man naturgemäß sprachfixiert. Und es wird mit dieser Sprachfixierung - des Gesprächs selbst im Sinne eines durch Gesprochen-Gehörtes dominierten Interaktionstyps, aber natürlich auch der Sprachfixierung der linguistischen Gesprächsforschung - zu tun haben, dass wir uns in der Gesprächslinguistik für den Raum bislang viel weniger interessiert haben als beispielsweise für die Zeit. Anders als die Zeitlichkeit des Gesprächs ist die Räumlichkeit des Gesprächs deshalb ein vernachlässigter Aspekt von Anwesenheit. Ob zu Recht, soll hier die Frage sein.

Mein Interesse an Räumlichkeit als Aspekt von Anwesenheit ist Teil eines größeren linguistischen Forschungsverbunds zum Zusammenhang von Sprache und Raum, der gleich im Anschluss kurz skizziert wird (siehe unten 2.). Ich werde mich dann ganz auf die interaktionstheoretischen Aspekte der Thematik konzentrieren und vorschlagen, mit der „Situierung“ eine auf Räumlichkeit bezogene Interaktionsaufgabe zu profilieren (siehe unten 3.). Mittel und Formen der Situierung sollen dann an einem Fallbeispiel aus dem Bereich der Kunstkommunikation illustriert werden: Es ist die Situation vor dem Kunstwerk, an der ich zeigen möchte, wie Räumlichkeit für Interaktion relevant wird und was man daran gesprächslinguistisch untersuchen kann (siehe unten 4.).

Das Ziel des vorliegenden Beitrags besteht gleichwohl nicht darin, eine gesprächslinguistische Untersuchung der behandelten Interaktionsepisoden vorzuführen. Auch geht es nicht darum, Ergebnisse einer empirischen Studie zur Interaktion in Kunstausstellungen vorzustellen. Sowohl zur gesprächslinguistischen Untersuchung raumbezogener Kooperation und Koordination als auch zur Analyse von Ausstellungskommunikation liegen solche empirischen Studien vor (auf die ich im Folgenden noch eingehen werde). Im vorliegenden Beitrag geht es vielmehr darum, einen theoretischmethodologischen Rahmen für die Thematisierung raumbezogener Interaktionsbeobachtungen zu skizzieren. An dieser Stelle, so meine ich, besteht angesichts einer schnell anwachsenden Zahl empirischer Detailanalysen ein konzeptioneller Nachholbedarf der Gesprächsforschung. 


\section{Sprache und Raum}

Die hier vorzustellenden Überlegungen verstehen sich als Beitrag zu einem größeren linguistischen Forschungsverbund, in dem es um das Verhältnis von Sprache und Raum geht. ${ }^{1}$

Wenn man keine Angst vor groben Vereinfachungen hat, kann man zwei Richtungen unterscheiden, aus denen man sich der Beziehung zwischen Sprache und Raum nähern kann:

So kann man z.B. sagen, dass Sprache grundsätzlich im Raum stattfindet. Mit „Sprache“ muss man dann den Gebrauch und die Verwendung von Sprache meinen, also so etwas wie Sprechen/Zuhören und Schreiben/Lesen. Sprachräume sind dann notgedrungen „Sprech-“ und „Zuhörräume“.2 Ein Sprech- und Zuhörraum kann etwas sehr Konkretes und sehr Kleines sein, z.B. eine Ecke in einem Zimmer. Er kann aber auch etwas sehr Abstraktes und flächenmäßig Großes sein, z.B. ein geographisches Areal. Es gibt viele und unterschiedliche linguistische Forschungstraditionen zu solchen Sprech- und Zuhörräumen: natürlich die Sprachgeographie und Dialektologie, natürlich die mikroanalytische Interaktionsforschung, aber auch die Soziolinguistik mit der Korrelation von Raumvariablen mit Sprachvariablen. Auch an die frühe Stadtsoziologie und Sozialgeographie könnte man in diesem Zusammenhang denken (Park 1926). Gerade angesichts dieser disparaten linguistischen und interdisziplinären Forschungstraditionen, die in der Regel mehr oder weniger isoliert voneinander gepflegt und aktualisiert werden (siehe aber unten!), ist es wichtig, sich klar zu machen, dass wir es in allen diesen Fällen mit „Sprech-“ und „Zuhörräumen“ zu tun haben, die nicht unabhängig vom Sprechen und Zuhören bestehen, sondern sich durch und mit dem Sprechen und Zuhören allererst konstituieren und zu einer sozialen Wirklichkeit werden, auf die man sich beziehen kann und die man - z.B. sprachwissenschaftlich - als diatopische Distribution objektivieren und kartographieren kann. Kein Sprechen und Zuhören ist davon ausgenommen, einen wie auch immer impliziten, häufig aber auch unüberhörbaren Bezug auf eine raumgebundene Zugehörigkeit zu artikulieren. „Mundarten“ sind dafür das vielleicht eindringlichste Beispiel.

\footnotetext{
1 Die Initiative zu diesem Forschungsverbund geht auf Gespräche mit meinen Zürcher Kolleginnen Elvira Glaser und Angelika Linke, auf Diskussionen mit Peter Auer und auf einen kontinuierlichen Austausch mit Wolfgang Kesselheim und Reinhold Schmitt zurück - vieles davon ist in diesen Beitrag eingeflossen, ohne dass ich das immer kenntlich machen kann.

2 Es gibt auch Schreib- und Leseräume: Auch das Schreiben und Lesen findet stets in Räumen statt, obwohl man sich die Schrift lange Zeit situations- und damit auch raumentbunden vorgestellt hat (vgl. kritisch dazu Peter Auer in diesem Band); textlinguistisch muss man entsprechend die Situation der Lektüre und die auf den Wahrnehmungsmöglichkeiten in einer Lektüresituation beruhenden Textualitätshinweise mit einbeziehen (Vorschläge dazu bei Hausendorf/Kesselheim 2008). Unter dem Stichwort „linguistic landscapes“ ist diese Räumlichkeit der Schrift neuerdings wieder vielfach ein Thema (vgl. Backhaus 2007; Scollon/Scollon 2003; Auer in diesem Band).
} 
Aus einer anderen Richtung kommend kann man sich auch für den Raum innerhalb der Sprache interessieren: für die Grammatik und das Lexikon, in denen Raum und Räumlichkeit eine Rolle spielen. Es geht dann, etwas zugespitzt, um den Raum in der Sprache und um die Erstarrungen, in denen sich Sprechräume in einer Sprache als Sprachräume niedergeschlagen haben. Mit dieser Erstarrung geht die oben bereits angedeutete Objektivierung und Repräsentierung (z.B. in Form der Kartographierung) von Räumen einher. Sprachräume können sich auf diese Weise vom Sprechen und Zuhören unabhängig machen - und ihrerseits dann sehr nachhaltig auf die Konstitution von Sprech- und Zuhörräumen einwirken. Unterschiedliche Sprachen haben, so ist gesagt worden, ihre jeweils eigene Weise, ihre Sprechräume zu kodieren, sie haben ihre jeweils eigenen ,grammars of space“ (Levinson/Wilkins (Hg.) 2006). Auch zu dieser Tradition gibt es eine Reihe von Arbeiten, z.B. aus der Typologie mit Arbeiten zur Deixis (Hickmann/Robert (Hg.) 2006), zur Grammatik und Semantik der Orts- und Raumreferenz (Vater 1991; Bernhard/Siller-Runggaldier 2008) und traditionell natürlich zur Toponymie bzw. Toponomastik.

Beide Zugangsweisen sind für sich genommen konzeptionell nicht recht überzeugend: Im einen Fall (Sprache im Raum) überlässt man den Raum mehr oder weniger den Geographen, den Architekten und Städteplanern und korreliert Räumliches mit Sprachlichem unterschiedlichster Art, wie es dem „correlational drive“ der frühen Soziolinguistik entspricht (Goffman 1964). Raum wird dabei als Kontext der Interaktion modelliert - für die Analyse ist er dann im Grunde verschenkt.

Im anderen Fall (Raum in der Sprache) reduziert man den Raum auf seine sprachlichen Reflexionen (in Grammatik und Lexikon), verbleibt ganz in der Sprache und erfasst das Sprachlose des Raumes gerade nicht. Räumlichkeit, könnte man übertreibend sagen, erscheint nurmehr als Fluchtpunkt sprachlicher Referenz und Lokalisierung, kaum aber als sprachlich und sensorisch-motorisch hervorgebrachte soziale Wirklichkeit (siehe aber unten!). So sehr unsere Raumwahrnehmung und unser Erleben und Behandeln von Raum durch Sprache gesteuert und geprägt sein mögen, so wenig lässt sich der Raum auf die Spuren seiner Reflexion in der Sprache reduzieren; die Räumlichkeit der sozialen Welt ist jedenfalls nicht auf Sprachlichkeit angewiesen, weder onto-, noch phylogenetisch. ${ }^{3}$

Es gibt in der Linguistik nach wie vor kaum Arbeiten, die beide Perspektiven miteinander verbinden. Aber es mehren sich Versuche, aus jeweils einer dieser Richtungen über die Brücke zu gehen, wenn man so sagen darf. Beispiele dafür gibt es etwa

3 Hinzuweisen wäre hier auf die „zoologische Evolution unseres Raumbezuges“, die wesentlich durch die „sensorische Ausstattung“ des Menschen und sein „sensorisches Dispositiv“ geprägt ist (Leroi-Gourhan 1980, S. 350 f.). 
- in der Arealtypologie, wenn man fragt, ob „Areal“ letztlich ein linguistischer oder geographischer Begriff ist (Bickel/Nichols 2006);

- in Beiträgen zu einer Ethnodialektologie und Ethnogeographie, die danach fragen, wie über Sprachräume auch außerhalb der wissenschaftlichen Dialektologie und Geographie gesprochen wird (Auer 2004; Jones et al. (2008); Schlottmann 2005);

- in der neueren Deixisforschung, die die „Sprechsituation“ mehr und mehr als eine sprachlich und gestisch hergestellte kognitive Wirklichkeit versteht und die sprachlichen Anteile an dieser Konstruktion zu bestimmen sucht (z.B. Hanks 1990; als Überblick jetzt Fricke 2007);

- oder auch in einer - oftmals typologisch orientierten - kognitiven Linguistik, die sich über Sprachräume einen Zugang zu so etwas wie „Raumkognition“ („spatial cognition“) verspricht (Levinson 1996; Herrmann/ Schweizer 1998; Hickmann/Robert 2006).

Dabei ist nicht zu übersehen, dass fast alle ambitionierten neueren Arbeiten zum Thema „Sprache und Raum“ sich letztlich für Kognition und Raum interessieren. Man braucht nur einen der vielen Sammelbände der letzten rund 15 Jahre aufzuschlagen, deren Titel mit den Bestimmungsstücken ,Sprache“ und „Raum“ operieren, um sich wie selbstverständlich in der kognitiven Linguistik wieder zu finden. ${ }^{4}$

Weitaus seltener findet man in der Linguistik Studien, die programmatisch nach dem Verhältnis von Kommunikation und Raum oder - wenn wir zunächst in der Welt des Sprechens und Hörens bleiben - nach dem Verhältnis von Interaktion und Raum fragen. Mit dieser Umfokussierung rückt naturgemäß die andere Nachbardisziplin der Linguistik in den Blick, also nicht die Psychologie (und ihre Spielarten), sondern die Soziologie. Hier gibt es einerseits eine ältere soziologische Tradition (insbesondere, aber nicht nur, verbunden mit den Arbeiten von Erving Goffman), die zumindest in Teilen der Linguistik gut bekannt ist. ${ }^{5}$ Andererseits gibt es auch eine neuere, seit einiger Zeit sehr lebhaft voran getriebene Soziologie des Raumes, die stärker kommunikationstheoretisch interessiert ist (vgl. z.B. Löw 2001; Dünne/Günzel (Hg.) 2006, Kap. IV; Schroer 2007; oder aus „,kultursoziologischer" Perspektive Richardson/Jensen 2003). Sie ist in der Linguistik noch so gut wie gar nicht rezipiert worden, und die Linguistik kommt ihrerseits in dieser neueren Raumsoziologie als Bezugsdisziplin so gut wie nicht vor. Das wäre ein eigenes Thema, auf das ich hier nicht weiter eingehen möchte. Im Folgenden soll stattdessen gezeigt werden, wie man ausgehend von einer interaktionstheoretischen Programmatik die skizzierte Dichotomie von Sprech- und Sprachräumen überwinden kann.

$4 \quad$ Vgl. dazu z.B. Bloom (Hg.) (1996); Pütz/Dirven (Hg.) (1996); Hickmann/Robert (Hg.) (2006); Levinson (2003); Tendenzen dazu auch schon in der etwas älteren Forschung zu Sprache und Raum (vgl. als Überblick z.B. Schweizer (Hg.) 1985).

5 Darauf komme ich noch zurück (siehe unten 4.). 


\section{Räumlichkeit als Aspekt von Anwesenheit}

Wenn man interaktionstheoretisch ansetzt, muss man Räumlichkeit als einen Aspekt von Anwesenheit konzipieren: In dem Moment, in dem Anwesenheit interaktiv hergestellt wird, also vereinfacht gesagt mit dem Blickkontakt des Von-Angesicht-zu-Angesicht (face-to-face), sind unweigerlich Personen, Zeit und Raum mitinbegriffen: Personen als ich und $d u$, als wir ${ }^{6}$ Zeit als ein jetzt, von dem aus prospektiv und retrospektiv Erwartungen manifestiert werden können, und Raum als ein hier, das all das umfasst an orts-, platz- und raumgebundener Umgebung, was gerade relevant sein soll für die laufende Interaktion. ${ }^{7}$ Räumlichkeit muss m.E. auf dieses „hier“ der Origo bezogen und als Teil der Sprechsituation (Bühler) konzipiert werden. Man muss sich dazu die Vielfalt und die Breite all dessen vor Augen halten, was in einem Gespräch ein ,hier" sein kann, also an all das denken, worauf sich Sprecher und Sprecherinnen in ihrem kommunikativen Alltag beziehen können, wenn sie den Ausdruck ,hier" verwenden - dann hat man sofort die ganze Welt möglicher Sprachräume im Sprech- und Zuhörraum! ${ }^{8}$

Als Aspekt von Anwesenheit muss Räumlichkeit - wie alles andere in der Sprechsituation auch - interaktiv hergestellt werden. Unabhängig davon, ob es einen Raum und ob es Räumlichkeit als physikalische Wirklichkeit gibt oder nicht geben mag (eine Frage für Physiker und Physikerinnen): Wenn ein Raum und wenn Räumlichkeit für die Interaktion relevant werden sollen, müssen wir beschreiben können, in welchen konkreten Erscheinungsformen der Interaktion sich diese interaktive Herstellung des Raumes vollzieht. Räumlichkeit ist, konversationsanalytisch gesagt, ein „interactive achievement", und nur als ein ,interactive achievement" ist Räumlichkeit für die Gesprächslinguistik von Bedeutung. Die Herausforderung besteht dann darin, diesen Gedanken auch konzeptionell und empirisch umzusetzen - also immer dann, wenn wir geneigt sind, doch wieder auf irgendeine Art von physikalischem Raum zurückgreifen zu wollen, Erscheinungsformen von Interaktion zu postulieren und nachzuweisen, in denen der Raum eben auch in seiner Physis für die Interaktion in Anspruch genommen

6 A. Schütz hat das bekanntlich zum Ausgangspunkt genommen, die „Gesichtsfeldbeziehung“ auch als „Wir-Beziehung“ zu kennzeichnen (Schütz 1972).

$7 \quad \mathrm{Ob}$ und in welcher Weise ein solches bier an die sinnliche Wahrnehmung der Anwesenden oder an ihre Erinnerung oder an ihre Vorstellungskraft appelliert, ist dabei noch nicht entschieden. Ein bier kann eben auch ein räumliches Setting sein, das sich die Anwesenden z.B. narrativ vergegenwärtigen (unabhängig davon, ob sie dabei auch den Ausdruck „hier“ verwenden). Vgl. dazu den Beitrag von Schmitt und Deppermann (in diesem Band).

8 In einer Spielerklärung beispielsweise kann ein „hier“ ein Punkt auf einem Spielbrett (das "Ziel“) sein: Und wo muss ich hin? hier, das ist das Ziel / / reigt auf Zielpunkt des Malefiz-Bretts/ / (Hausendorf 2003, S. 253). In einem Gespräch an einem Bücherstand in Ostberlin kann ein „hier" ein politisch-geographisch definiertes Territorium (das der DDR) sein: der / zeigt auf ausliegendes Buch von Chomsky// war bier ja lange verpönt ne? (Hausendorf 2000, S. 188). 
wird. ${ }^{9}$ Der Vorschlag, der in diesem Beitrag vorgestellt werden soll, besteht darin, eine für Interaktion konstitutive Aufgabe zu postulieren, die sich genau aus dieser Anforderung der Herstellung von Räumlichkeit ergibt. Diese Aufgabe soll hier „Situierung“" genannt werden.

\section{Situierung als Interaktionsaufgabe}

Wer von „Situierung“ als Interaktionsaufgabe spricht, darf von anderen konstitutiven Interaktionsaufgaben nicht schweigen. In und mit Interaktion werden unterschiedliche Interaktionsaufgaben gleichzeitig gelöst - eine Herausforderung für interaktionsanalytische Studien jedweder Couleur. Interaktion als Problem- und Aufgabenlösung zu sehen, ist konzeptionell nicht neu. In theoretischer Perspektive umrissen wird sie etwa in Goffmans Einleitung zur „frame analysis“ (Goffman 1977). In analytischer Perspektive entspricht sie vor allem der Grundvorstellung der frühen Konversationsanalyse (Gesprächsbeendigung als konversationelles „Problem“: Schegloff/ Sacks 1973). Konversationsanalytische Studien operieren deshalb durchgängig mit der Idee konversationeller Probleme und/oder Aufgaben (vgl. dazu z.B. die in Hausendorf 2007 (Hg.) veröffentlichten Beiträge). Eine übergreifende Modellierung der für Interaktion allgemein konstitutiven Aufgaben wird gleichwohl nur selten versucht. Konzeptionell wegweisend ist hier nach wie vor die frühe Einführung in die ethnomethodologische Konversationsanalyse von Kallmeyer und Schütze (Kallmeyer/Schütze 1976). Sie hat in mancherlei Hinsicht vorweggenommen, was inzwischen auf empirische Weise in zahllosen Beiträgen zur Gesprächsforschung erarbeitet, differenziert und weiter entwickelt worden ist. Genügend Gründe also, um eine solche Modellierung der für Interaktion konstitutiven Aufgaben wieder in Angriff zu nehmen.

Die folgende Darstellung, die einen bereits an anderer Stelle publizierten Gedanken wieder aufnimmt (Hausendorf 2007), geht davon aus, dass Interaktion als soziales System (im Sinne Goffmans, aber auch im Sinne der Luhmannschen Systemtheorie: Hausendorf 1992) verstanden werden kann. Dass dieses Interaktionssystem ein multi-tasking system ist, gehört zu den Grundeinsichten der letzten rund 35 Jahre linguistischer Gesprächsforschung; wenn wir nach den einzelnen Aufgaben fragen, die in und mit Interaktion gleichzeitig bearbeitet werden, stoßen wir sofort auf lauter alte Bekannte, auf die üblichen Verdächtigen der Konversations- und Interaktionsanalyse. Das folgende Schaubild bringt diese gut bekannten Interaktionsaufgaben in Form von Fragen zum Ausdruck:

$9 \quad$ Wir werden sehen, dass dieser Gedanke vor allem dazu zwingt, Wahrnehmung (in der ganzen Bandbreite menschlicher Sensorik) und Bewegung (in der ganzen Bandbreite menschlicher Motorik) als Erscheinungsformen von Interaktion auffassen zu können (siehe unten 5.). 


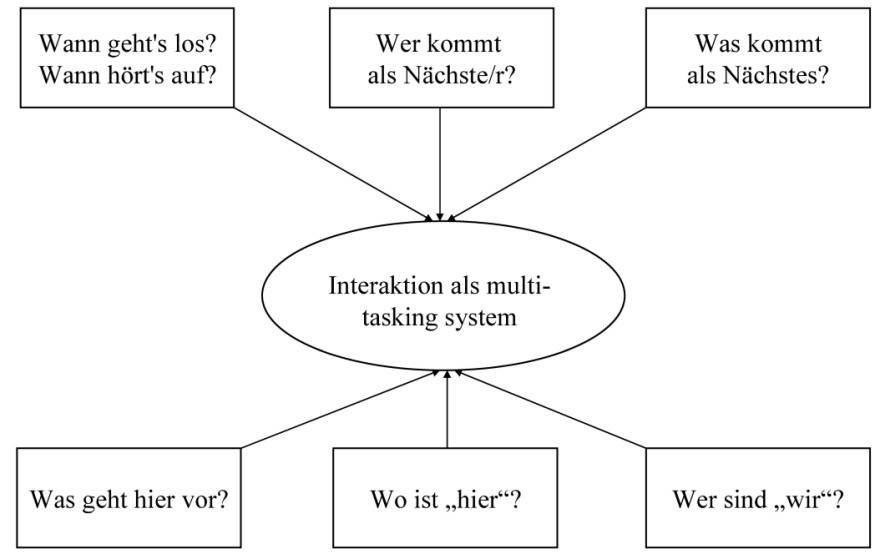

Abb. 1: Interaktion als multi-tasking

Hinter diesen Fragen stehen jeweils viel beschriebene interaktive Aufgaben:

- die Herstellung, Aufrechterhaltung und Auflösung von Anwesenheit (Wann geht's los? Wann hört's auf?),

- der Sprecherwechsel (Wer kommt als Nächster?),

- die Organisation von Beiträgen und Themen (Was kommt als Nächstes?),

- die Kontextualisierung und Rahmung des Geschehens (Was geht hier vor?),

- die Selbst- und Fremddarstellung (Wer sind „wir“?) und eben auch

- die Situierung des Geschehens (Wo ist „hier"?).

Es ist hier nicht der Ort, die Anzahl und Benennung dieser Aufgaben zu diskutieren. ${ }^{10}$ Behauptet werden soll aber, dass es sich bei diesen Aufgaben tatsächlich um interaktionskonstitutive Aufgaben handelt, die also immer irgendwie bearbeitet (,gelöst") werden müssen. Behauptet werden soll weiterhin, dass die Bearbeitung dieser Aufgaben jeweils im interaktiven Hinter- oder Vordergrund geschehen kann, woraus sich wie von selbst eine Typologie unterschiedlicher Interaktionsereignisse ergibt. Wichtig(er) für den vorstehenden Zusammenhang ist die Frage, wo und wie Raum und Räumlichkeit in diesem Aufgabenszenario auftauchen.

Zunächst kann man leicht sehen, dass Raum und Räumlichkeit offenkundig auch für andere Interaktionsaufgaben relevant sind: Der Raum kann beispielsweise auch zum Thema von Beiträgen werden, zu dem also, worü-

$\overline{10} \quad$ Ein Indiz für die Angemessenheit dieses Modells sehe ich darin, dass man es gut zum Ausgangspunkt nehmen könnte, eine Einführung in die Gesprächslinguistik zu verfassen: Maßgebliche Ergebnisse gesprächslinguistischer Forschung ließen sich ohne große Mühe entlang dieser Aufgaben präsentieren (vgl. dazu die gerade erschienene Einführung in die Konversationsanalyse von E. Gülich und L. Mondada (2008), die diesen Eindruck m.E. sehr anschaulich belegt). 
ber man spricht und zu dem, was man sprachlich und gestisch-spielend in Szene setzen kann, z.B. beim Erzählen (Aufgabe: Themen- und Beitragsorganisation; vgl. dazu den Beitrag von Schmitt und Deppermann im vorliegenden Band); Raum kann auch zur Zugehörigkeitsressource werden, also zur „Verortung“ der TeilnehmerInnen genutzt werden (Aufgabe: Selbstund Fremddarstellung; vgl. dazu z.B. die Relevanz von Herkunftsprädikaten für die Klärung von Zugehörigkeit: Hausendorf 2000, S. 197 ff.), er trägt in vielen Fällen maßgeblich zum Sprecherwechsel, aber auch zur Rahmung bei. ${ }^{11}$ Diese Relevanz von Raum und Räumlichkeit auch für die anderen Interaktionsaufgaben ist konzeptionell dadurch erfasst, dass die Bearbeitung der unterschiedlichen Interaktionsaufgaben grundsätzlich gleichzeitig erfolgt, sich also empirisch sehr funktionale Überlappungen zwischen den Aufgaben und ihrer Bewältigung ergeben.

Die Übersicht (siehe oben Abb. 1) lässt des Weiteren erkennen, dass die Situierung als eigenständige Interaktionsaufgabe in der Konversationsanalyse und Gesprächsforschung bislang weit weniger Aufmerksamkeit gefunden hat als die anderen Aufgaben. Wenn man z.B. sieht, wie die naturgemäß sehr prominenten „Wer/Was kommt als Nächster/s“-Fragen ${ }^{12}$ dazu geführt haben, Zeitlichkeit im Sinne von Sequentialität zu einem herausragenden Konzept zu entwickeln, kann man auch sagen: Räumlichkeit ist als Aspekt von Anwesenheit bislang fast noch ein blinder Fleck.

Diese Diagnose gilt auch dann, wenn man weiss, dass es eine sehr ehrwürdige sozialwissenschaftliche Tradition gibt, die unter dem etwas unglücklichen Titel „Context Analysis“ bekannt geworden ist und die von Bateson über Birdwhistell bis Kendon reicht (um nur drei Namen zu nennen) und in der Situierung immer ein Thema war. ${ }^{13}$ Wenn diese und verwandte Traditionen der sozialwissenschaftlichen Interaktionsanalyse heute an vielen Stellen aufgenommen und wieder belebt werden (vgl. Schmitt 2007), dann zeigt sich darin eben auch, wie lange wir in der Gesprächsforschung die Situierung als Interaktionsaufgabe - und mit ihr den Raum - mehr oder weniger vergessen hatten. ${ }^{14}$

$11 \quad$ Im Falle der Rahmung ist die Überschneidung mit der Situierung besonders deutlich. Wir werden deshalb darauf noch zurückkommen (siehe unten 6.).

12 „The practical question par excellence, "What to do next?”' (Garfinkel 1967, S. 12); „What's next?" (Schegloff/Sacks 1973).

13 Hinweise auf diese Tradition finden sich z.B. bei Kendon (1990, S. 15 ff. („Some context for context analysis: a view of the origins of structural studies of face-to-face interaction")); vgl. zusammenfassend auch Knoblauch (2005, S. 267 f.).

14 Neben den Arbeiten zur „Multimodalität der Interaktion“ (Schmitt 2006; Norris 2004) wären hier auch die work-place-studies zu nennen, aus denen in den letzten Jahren viele neue Impulse gekommen sind (z.B. Heath/Hindmarsh 2000; Hindmarsh/Heath 2000). „Situatedness“, „Embodiment“, „Multimodality“ und „Discourses in Place“ sind im Grunde neue Stichworte, mit denen die Relevanz der Aufgabe der Situierung aus jeweils unterschiedlichen Perspektiven und mit unterschiedlichen Gewichtungen in den letzten Jahren hervorgehoben worden ist (vgl. z.B. Goodwin 2000; Frank et al. (Hg.) 2008; Scollon/Scollon 2003; Überblick z.B. bei Mondada 2007). 
Wenn man sich der Situierung zuwendet, ist zunächst nicht zu übersehen, dass - wie Goffman formuliert - „das natürliche Zuhause von Sprache" eines ist, in dem die Sprache nicht immer gegenwärtig ist. ${ }^{15}$ Die an den Anfang dieses Beitrags gestellten Beispiele aus dem Sport (das Fußballspiel), aber auch aus dem Krankenhaus (Operation) liefern dafür sehr anschauliche Belege. Situierung lebt entsprechend, das werde ich im empirischen Teil zu zeigen versuchen (siehe unten 5.), zunächst von der Ausnutzung von Anwesenheit im Vollsinne der Gegenwart von Menschen: also von der Sensorik, der Motorik und der Kognition Anwesender. Der Sprechund Zuhörraum ist insofern immer auch ein Wahrnehmungs-, ein Bewegungs- und ein Handlungsraum, der als solcher nicht auf Sprechen und Zuhören, wohl aber auf Anwesenheit angewiesen ist.

Wenn und sobald gesprochen und zugehört wird (und das ist oft genug der Fall), kann die Situierung dann natürlich auch auf Sprache zurückgreifen: auf Deixis in einem engeren Sinne (siehe unten 5.), auf die Grammatikalisierung räumlicher Verhältnisse im weitesten Sinne und auf die Lexik und Semantik möglicher Räume, Plätze und Orte. Es sind dies ja letztlich nichts anderes als Erstarrungen und Verfestigungen, sprachlich routinisierte Lösungen kommunikativ-interaktiver Situierungsaktivitäten. Das gilt diachron wie synchron, typologisch wie einzelsprachlich und phylogenetisch wie ontogenetisch. Empirisch fällt die Konstitution von Sprech- und Zuhörräumen deshalb mit der Konstitution von Sprachräumen zusammen (so sehr sich auch die Untersuchung der Phänomene disziplinär und interdisziplinär unterscheiden mag, siehe oben 2).

Schließlich wird sich zeigen, dass man sich den Raum selbst nicht als tabula rasa vorstellen sollte. Es gibt, etwas zugespitzt, eine genuine Lesbarkeit des Raumes (Kesselheim/Hausendorf 2007), und die interaktive Lösung der Situierungsaufgabe wäre ohne solche Anschlussmöglichkeiten im Raum oft gar nicht denkbar. Wenn wir uns für Situierung interessieren, dürfen wir also solche nicht interaktiven, also nicht an Kopräsenz gebundenen, sondern kommunikativ-dauerhaften Konstitutionen von Räumlichkeit nicht übersehen. In ihnen vor allem vollzieht sich hintergründig die gesellschaftliche Rahmung von Interaktion (siehe unten 6.).

Anwesenheit, Sprachlichkeit und die Lesbarkeit des Raumes sind die wesentlichen Ressourcen, auf die die Situierung zurückgreifen kann, wie das folgende Schaubild vorausgreifend zum Ausdruck bringt:

15 „Note then that the natural home of speech is one in which speech is not always present.“ (Goffman 1964, S. 135). Goffman meint mit dem „natürlichen Zuhause“ der Sprache die soziale Situation der face-to-face Interaktion, wie sie über eine lange Zeit als eigenständiger Gegenstand mehr oder weniger ignoriert worden ist. 


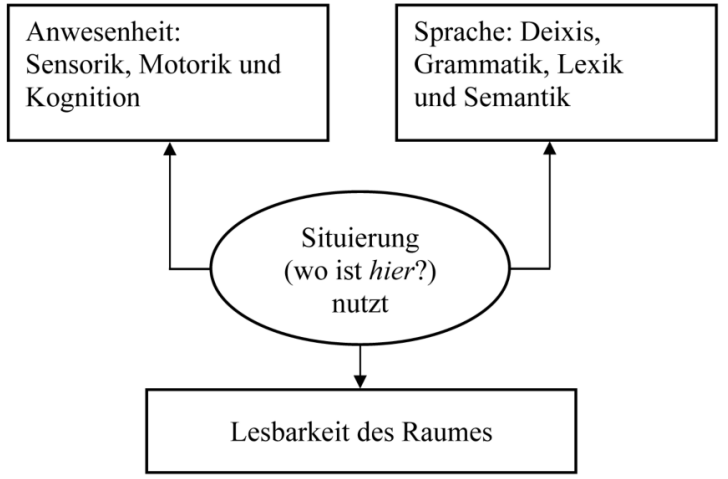

Abb. 2: Ressourcen der Situierung

Empirisch tut sich an dieser Stelle ein großes und weites Feld auf, das die Linguistik bislang nur sehr ausschnittweise betreten hat (siehe oben) - und das sie in theoretisch-konzeptioneller Hinsicht, wenn man z.B. an den „spatial“ oder „topographical turn" in den Kulturwissenschaften denkt (Döring/Thielmann (Hg.) 2008; Schroer 2008; Schlögel 2003, S. 60-71; Weigel 2002), weitgehend anderen Disziplinen überlassen hat. Auch das wäre ein eigenes Thema.

Mit dem folgenden Fallbeispiel werde ich vor allem das Nutzen von Anwesenheit in seinen senso-motorischen Aspekten zu illustrieren versuchen. ${ }^{16}$ Den Bereich des Sprachlichen, aber auch die Lesbarkeit des Raumes werde ich dabei nur andeuten, nicht aber systematisch erörtern können. Das Fallbeispiel soll, wie eingangs betont, der Illustration eines Forschungsrahmens dienen, nicht der empirischen Analyse der Details der Raumkonstitution.

\section{Situierung vor dem Kunstwerk}

Im Mittelpunkt der folgenden Beobachtungen steht eine alltägliche Szene: Ein paar Leute gehen zusammen in eine Ausstellung. Was so einfach und so trivial klingt, ist gleichwohl Moment einer ausgewachsenen sozialen Praxis:

$16 \quad$ Wie die Räumlichkeit sind auch die senso-motorischen Grundlagen der Interaktion bislang stark vernachlässigt worden - obwohl es insbesondere in der phänomenologischen Tradition viele Anknüpfungspunkte gibt, an die die linguistische Gesprächsforschung anschließen könnte. Vgl. dazu die theoretische Diskussion der Thematik bei Loenhoff (2002), die mit den hier vorzustellenden empirischen Beobachtungen ausgesprochen kompatibel scheint. Loenhoff geht bei seinen Überlegungen nicht von Räumlichkeit aus, sondern von der Art und Weise, wie Kommunikationstheorien Körperlichkeit zu thematisieren gestatten, kommt dabei dann aber auch auf Räumlichkeit zu sprechen (speziell ebd., S. 53 ff.: „Der Körper und die situative Räumlichkeit"). 
der sozialen Praxis des gemeinsamen Ausstellungsbesuchs. Ich werde zu zeigen versuchen, dass diese Praxis eine Vielzahl von Situierungsaktivitäten verlangt. Die Welt der Ausstellungen ist deshalb für unser Thema besonders geeignet - was andere auch schon vor mir bemerkt haben (z.B. Kesselheim 2009; z.B. Vom Lehn/Heath 2007).

Der Videoausschnitt, den ich im Folgenden besprechen werde, ${ }^{17}$ beginnt damit, dass Claudia und Melanie einen Ausstellungsraum betreten haben und gemeinsam auf ein am Boden befindliches Objekt zusteuern, sich dann hinunterbeugen und - mit dem ins Bild kommenden Nils - um das Objekt herum hockend verweilen:

Ausschnitt 1: öbis zwüsche bimbeermarmelade und bluet ${ }^{18}$

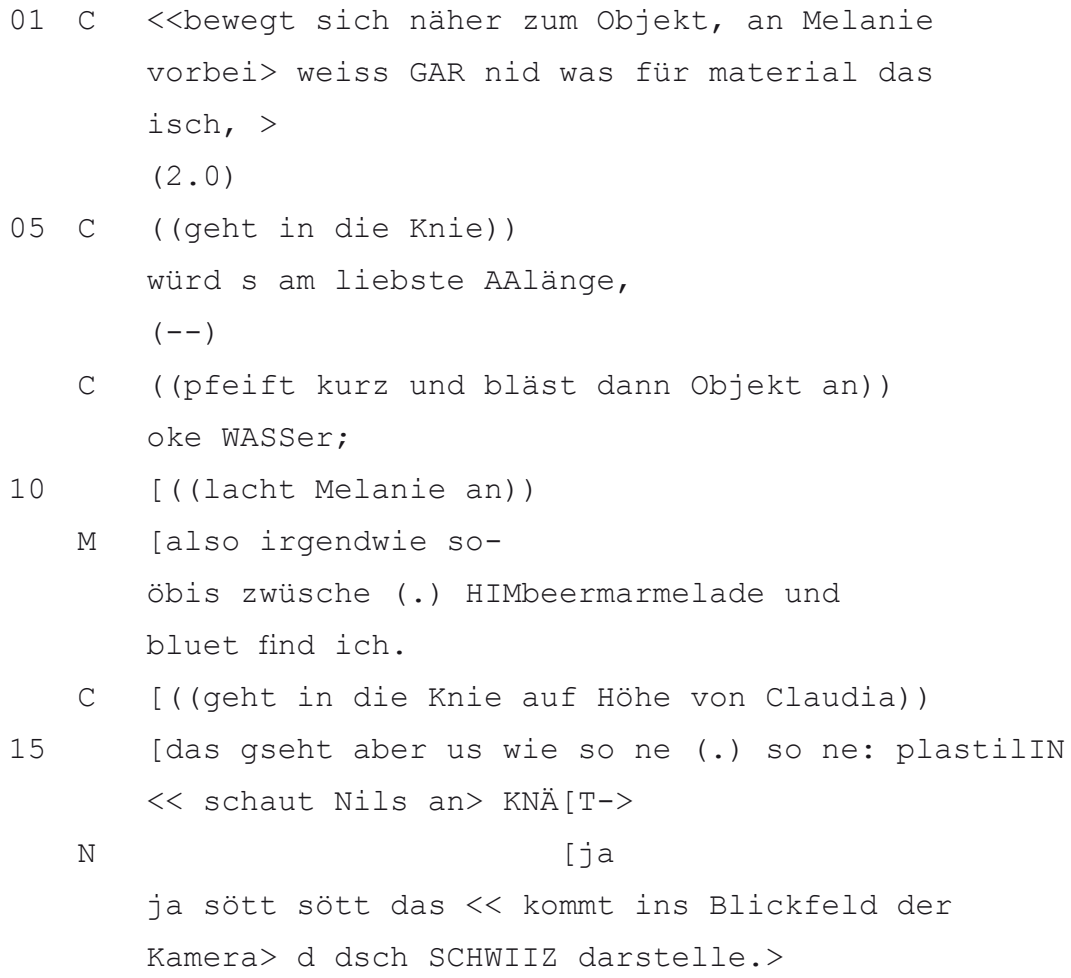

$17 \quad$ Ich verdanke das Beispielmaterial Sabina Gröner, die es im Rahmen einer Lehrveranstaltung („Kunstgespräche“, Zürich, FS 2007) erhoben, transkribiert und analysiert hat. Den an der Szene beteiligten Personen sei für die Verwendung und den Abdruck der Standbilder gedankt!

18 Transkription: Sabina Gröner. Transkriptionszeichen in Anlehnung an GAT, grobe Wiedergabe mundartlicher Besonderheiten des Schweizerdeutschen mit literaler Umschrift. 
Keine spektakuläre, vielmehr alltägliche Szene aus einem gemeinsamen Ausstellungsbesuch. Im Folgenden interessiert, wie in diesem Ausschnitt „Raum“ und „Räumlichkeit" ins Spiel kommen. Ich werde den Raum dazu nach und nach als Wahrnehmungs- (5.1), Bewegungs- (5.2), Handlungs- (5.3) und Spielraum (5.4) vorstellen.

\subsection{Wahrnehmung}

Für die soziale Praxis des gemeinsamen Ausstellungsbesuchs, der wir gerade beiwohnen, ist zunächst und offenkundig die sinnliche Wahrnehmung, insbesondere die visuelle Wahrnehmung, ist das Sehen der Anwesenden von besonderer Bedeutung. Der Raum ist, das lässt sogar noch die sprachfixierte Transkription erkennen, für die Interaktion neben allem anderen, was er noch sein mag, ein Wahrnehmungsraum. Es geht dann darum, aus der Fülle an Möglichkeiten des Wahrnehmbaren die für die Interaktion gerade relevanten Wahrnehmungen auszuwählen und interaktiv zu etablieren. Dafür gibt es unter Anwesenden nur einen Weg: die fraglichen Wahrnehmungen müssen selbst wahrnehmbar (gemacht) werden. Derartige „Wahrnehmungswahrnehmungen " (Luhmann) sind für Interaktionsforscher und Interaktionsforscherinnen nichts Ungewöhnliches. Schon der Blickkontakt ist ja genau das: Indem mit dem Blickkontakt in der Regel wahrgenommen werden kann, dass wahrgenommen wird, erzeugt er in einer Art Kurzschluss „Anwesenheit" als Interaktionsbedingung. ${ }^{19}$ Das erfasst zunächst und primär die Anwesenden in ihrer Kopräsenz, also das „wir“. Für das „hier“ müssen dann andere Phänomene aufkommen, die garantieren, dass das Sehen von etwas Drittem (das nicht der Andere ist) selbst sichtbar wird. Ich greife dazu zwei bekannte Phänomene heraus.

Das erste für die Etablierung gemeinsam geteilter Aufmerksamkeit relevante Phänomen zeigt sich schon in der Annäherung an das Objekt:

19 Ich greife an dieser Stelle auf Vorarbeiten interaktionssoziologischer (Goffman 1964) und systemtheoretischer (Luhmann 1984, S. 560 ff.) Provenienz zurück. In beiden Traditionen wird übereinstimmend die Relevanz von Wahrnehmungswahrnehmungen für die Emergenz von Interaktion betont (vgl. Hausendorf 1992). 
Ausschnitt 2: was für material das isch

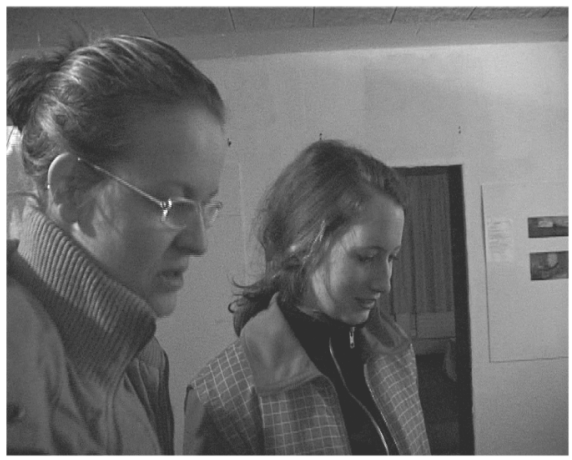

01 C <<bewegt sich näher zum Objekt, an Melanie vorbei> weiss GAR nid was für material das isch, > $(2.0)$

Das Bild in Ausschnitt 2 illustriert die Synchronisierung der Ausrichtung der Wahrnehmungsorgane (Blickrichtung und Köperzuwendung) von Claudia und Melanie: Dadurch, dass Claudia und Melanie in die gleiche Richtung schauen und ihre Sinnes- bzw. Kommunikationsorgane auf ein gleiches Ziel ausrichten, wird die Wahrnehmung von etwas „,vorne unten“ selbst wahrnehmbar. Das Sehen wird sozusagen sichtbar gemacht. Man könnte das Ko-Orientierung nennen (siehe unten).

Noch deutlicher zeigt diesen Mechanismus der folgende Ausschnitt, der einsetzt, nachdem die Beteiligten um das Objekt herum in die Knie gegangen sind:

Ausschnitt 3: schwiizerkrüz

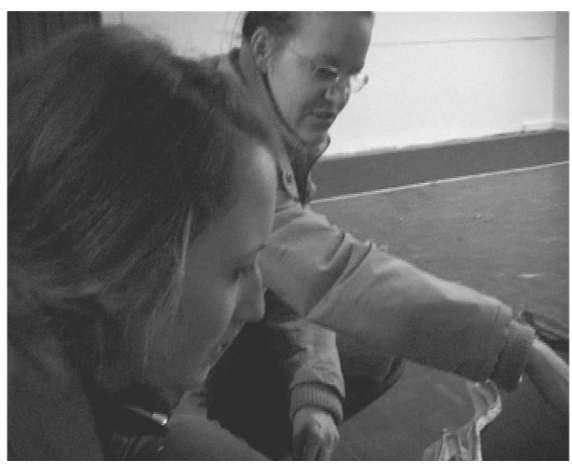

N ja sött sött das $<<$ kommt ins Blickfeld der

Kamera> d dsch SCHWIIZ darstelle.> 
Ausschnitt 3 zeigt ein Beispiel für verbale und nonverbale Deixis (Demonstrativum und Zeigegeste): Deixis ist - eng gefasst (Hausendorf 2003) - nichts Anderes als das Sichtbarmachen des Sehens, ${ }^{20}$ die visuelle Wahrnehmung eines bestimmten Details wird selbst unmissverständlich wahrnehmbar gemacht. Der ausgestreckte Arm mit dem ausgestreckten Finger fungiert gewissermaßen als verlängerter Blick. Unterstützt wird das hier - wie so oft - durch Sprache, durch das Demonstrativum „das“, das wie ein „shifter" (Jakobson) dazu dient, auf Wahrnehmung als relevante Interaktionsmodalität gleichsam umzuschalten. Der Hinweis, dass Wahrnehmung wahrgenommen werden soll, ist der Sinn der demonstratio ad oculos et ad aures (Bühler 1982). Mit „Zeigen“ oder „Lokalisieren“ hat das nicht viel zu tun (vgl. dazu auch de Mulder 1996).

Wenn in diesem Beitrag von Deixis die Rede ist, ist immer dieser Spezialfall gemeint, der nach Weinrich (1993, S. 444 f.) am Rande der Sprache steht und ein Signal an der Grenze zwischen Code und Situation ist. Das gilt nicht für alle Sprachzeichen, die landläufig unter dem Sammelbegriff „Deixis" subsumiert werden. Nur dann, wenn ein mit Anwesenheit grundsätzlich unterstellbares Minimum an geteilter Aufmerksamkeit zugunsten von Spezialwahrnehmungen in irgendeiner Hinsicht expandiert werden soll, sind deiktische Hinweise im oben erläuterten engeren Sinne notwendig. Wahrnehmung kann auch ohne Deixis - z.B. durch die Verwendung der verba sentiendi - wahrnehmbar gemacht werden, aber mit und durch Deixis geschieht das unvergleichlich ökonomisch, unvergleichlich effektiv und unvergleichlich unscheinbar.

Synchronisation und Deixis sind zwei herausragende Phänomene, die illustrieren, wie im Medium von Wahrnehmungswahrnehmung z.B. Objekte im Raum interaktiv relevant gemacht und in diesem Sinne tatsächlich „hergestellt" werden können.

20 Dieses Verständnis von Deixis ist natürlich begründungspflichtig. Es schließt eng an Bühlers Konzeption an (Hausendorf 2003). Für die Argumentation im vorliegenden Beitrag muss es aber nicht vorausgesetzt werden. 
Wenn der Raum stets ein Wahrnehmungspotential für die Sinnesorgane der Anwesenden darstellt, ergibt sich daraus eine Daueranforderung für die Interaktion: Die für die Interaktion gerade relevanten Wahrnehmungen im Raum müssen ausgewählt und etabliert werden. Ich nenne das „Ko-Orientierung". Ko-Orientierung vollzieht sich in Relation zu den Sinnesorganen der Anwesenden: oben und unten, vorne und hinten, rechts und links Ausdrücke wie diese liefern Beispiele für Orientierungen, wie sie für die Navigation im Nahbereich der Interaktion unerlässlich sind. Die Ko-Orientierung greift als Aspekt der Situierung vor allem auf die Sensorik der Anwesenden zurück. Man kann an diesem Feld anschaulich machen, was es heißt, dass die Anwesenden zu den "Sensoren des Interaktionssystems“ werden (Luhmann 1984, S. 558). Ohne Ko-Orientierung wäre die soziale Praxis eines gemeinsamen Ausstellungsbesuchs undenkbar.

Nicht immer, aber sehr häufig sind Ko-Orientierungen der Ausgangspunkt für Konfigurationen, die Bewegung implizieren.

\subsection{Bewegung}

Für die soziale Praxis des Ausstellungsbesuches ist neben der Wahrnehmung unübersehbar auch die Bewegung der Anwesenden relevant, wenn man so will: die Motorik und die Kinesik der Navigation im Ausstellungsraum. Das „hier“ erscheint in dieser Hinsicht nicht nur als Wahrnehmungs-, sondern auch als Bewegungsraum. ${ }^{21}$ Stärker noch als das Wahrnehmungspotential von Räumlichkeit bedroht ihr Bewegungspotential die Interaktion akut: Anwesende können durch Bewegung im Raum jederzeit Abwesenheit wählen! Bei Bewegung der Anwesenden ist Interaktion also ständig durch Selbstauflösung bedroht. Das Problem ist dann - wenn man nicht gerade zusammen sitzt -, die Interaktion trotz und bei Bewegung aufrechtzuerhalten, vereinfacht gesagt: zusammen zu bleiben. In einer Ausstellung ist das ein vertrautes Problem: Es soll nicht jeder für sich die Ausstellung besuchen, sondern die TeilnehmerInnen sollen gemeinsam die Ausstellung besuchen. Man muss also Konfigurationen finden, die den Ausstellungsbesuch immer wieder als gemeinsames Ereignis aufrechterhalten, erfahrbar und erlebbar machen. ${ }^{22}$

$21 \quad$ In einer konkreten Situation wie der hier besprochenen fallen der Wahrnehmungs- und der Bewegungsraum empirisch mehr oder weniger zusammen. Gleichwohl scheint mir die Unterscheidung von Wahrnehmung und Bewegung analytisch ausgesprochen fruchtbar, weil die auf Sensorik beruhende Herstellung von Raum und Räumlichkeit von der auf Motorik beruhenden Herstellung von Raum und Räumlichkeit unterschieden werden kann und muss. $\mathrm{Ob}$ und in welcher Weise Bewegung Wahrnehmung impliziert und Wahrnehmung umgekehrt Bewegung impliziert, ist dafür nicht ausschlaggebend.

22 Von „Konfigurationen“ ist in unmittelbar vergleichbarem Zusammenhang auch bei Vom Lehn/Heath (2007) die Rede. Bei Kendon (1990) heißt es mit ähnlicher Bedeutung auch „spatial positioning“. 
Eine typische, für die soziale Praxis des gemeinsamen Ausstellungsbesuchs zentrale Konfiguration ist das gemeinsame Verweilen vor dem Kunstwerk. Ausschnitt 1 zeigt den Beginn dieser Konfiguration, der folgende Ausschnitt zeigt ihre Aufrechterhaltung und Auflösung:

Ausschnitt 4: ich glaub es isch jugoslawie lueg (Fortsetzung von Ausschnitt 1)

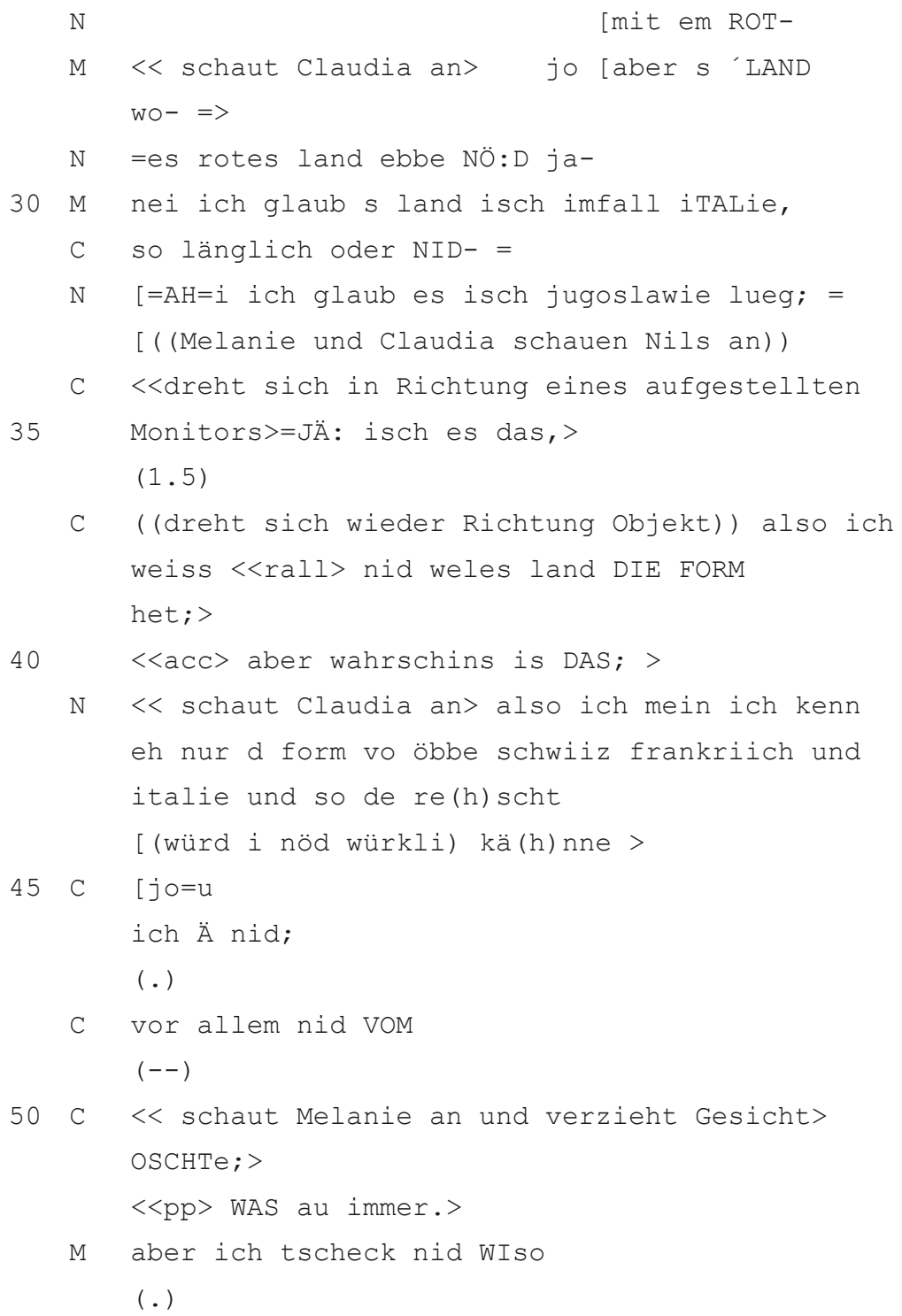

(.) 
55 M ah weisch WIso;

$(-)$

M jo ich glaub es isch scho $<<$ h $>$ irgendwie $>$ BLUET $<<t>$ aso->=

C $=j \circ \mathrm{s} s$ het öbis BLUE

60 [tigs; (verzieht kurz Gesicht)

M [d SCHWIIZ wo irgendwie öbis bluetigs <<steht auf> in däm LAND irgendwie het> oder so.=

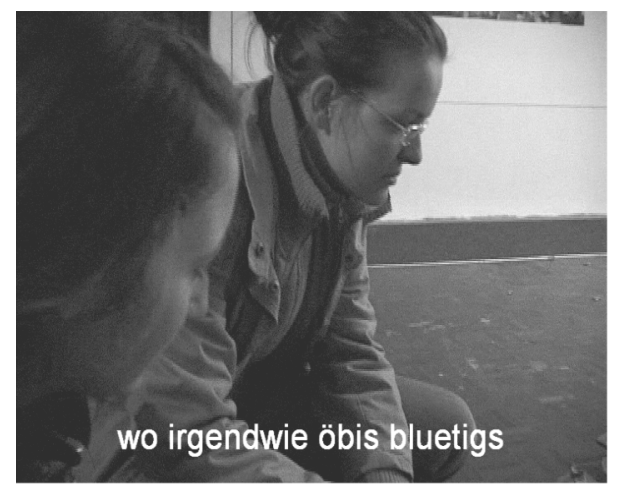

Z. 61

$\mathrm{C}=j \circ \mathrm{s} \mathrm{s}$ het öbis BLUE

60 [tigs; (verzieht kurz Gesicht)

M [d SCHWIIZ wo irgendwie öbis bluetigs $<<$ steht auf> in däm LAND irgendwie het> oder so.=

$\mathrm{N}=$ ja döte

$65 \mathrm{C} \quad<$ steht auf und verzieht Gesicht $><<$ p $>$ KEI ahn $[$ ig. $>>$

$\mathrm{N}$ <<steht auf> [ebbe halt> ähm HAja oke:; (2.5)

C $\quad<$ schaut Melanie an> aber d finger hän si jo 70 nid im SPIIL ka; $(--)$

C WäHRend em krieg oder $<<$ hebt Schultern an $>$ WEISCH->>= =ich glaub $\mathrm{s}$ si=ch ich WEISS es nid aber 

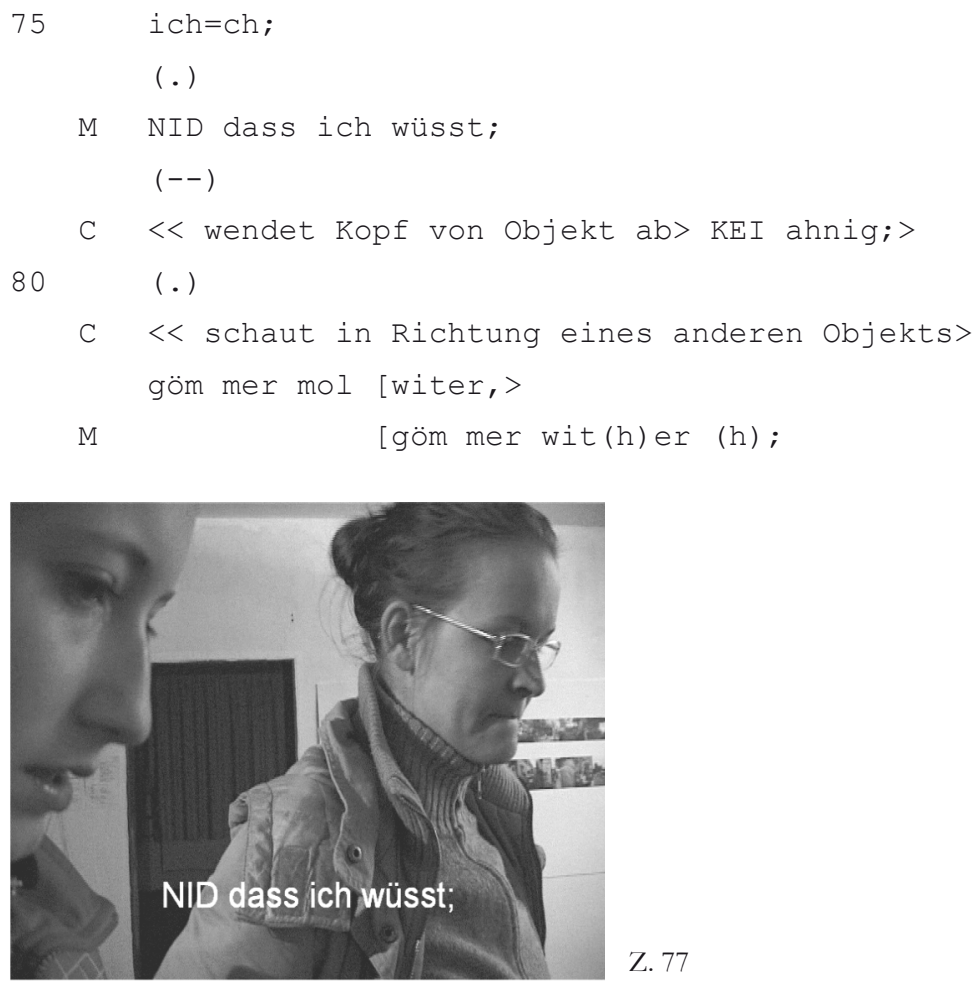

Ungeachtet ihrer großen Selbstverständlichkeit fallen Konfigurationen wie das hier illustrierte gemeinsame Verweilen vor dem Kunstwerk nicht vom Himmel, sondern müssen hergestellt, aufrechterhalten und auch wieder aufgelöst werden. Das erfordert spezielle Koordinierungsaktivitäten. Man kann sie am vorstehenden Ausschnitt leicht anschaulich machen, weil die fragliche Konfiguration in diesem Fall (das Wahrnehmungsobjekt befindet sich auf dem Boden) motorisch und kinetisch anspruchsvoll ist. Wir wollen uns im Folgenden ganz auf die Auflösung der Konfiguration beschränken (siehe oben Ausschnitt 4, etwa ab Z. 56). Je mehr diese Auflösung als eine synchronisierte Bewegung ausgeführt wird, desto weniger bedroht sie offenkundig die Interaktion. Synchron ausgeführte Bewegungen, in diesem Fall: das gleichzeitige Aufstehen und Weggehen, erfordern eine anspruchsvolle Choreographie, die nicht umstandslos vorausgesetzt werden kann. Typischerweise kommt es in solchen Fällen im Alltag zu Verzögerungen und Verlangsamungen, häufig auch zu unmittelbaren Folgebewegungen. So folgen Claudia und Nils im vorliegenden Fall mehr oder weniger synchron Claudia in ihrer Aufstehbewegung und Wegorientierung: 
Ausschnitt (5): Gemeinsames Aufstehen in zwei Etappen

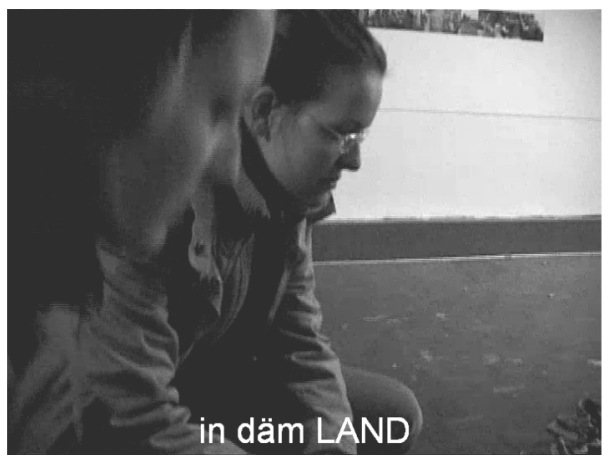

Z. 62: Melanie (links im Bild) während des Aufstehens

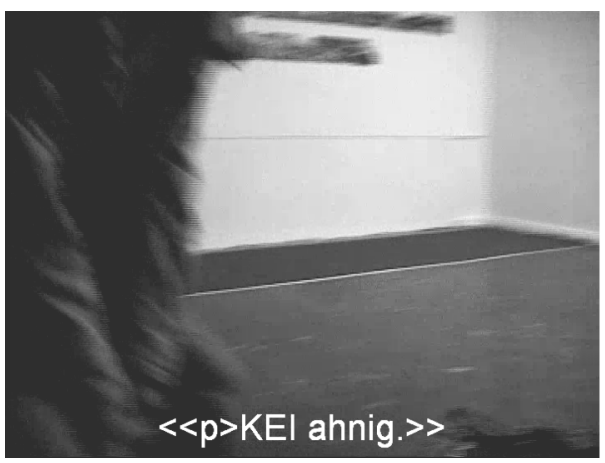

Z. 65-66: Claudia und Nils (nicht im Bild) folgen Melanies Aufstehbewegung

Folgebewegungen wie diese hat Goffman als Beispiele für typisches Gruppen- bzw. „Rudel“"verhalten beschrieben:

Tierische soziale Gruppen - Herden, Rudel, Scharen, Banden, Meuten, Schwärme - zeigen die besondere Eigenschaft, daß die Mitglieder jeder dieser Gruppen gewöhnlich in gegenseitiger Wahrnehmungsreichweite bleiben. (Goffman 1974, S. 19)

Typischerweise werden Auflösungen von Konfigurationen im Alltag (also nicht in professionell herbei- und ausgeführten Konstellationen wie etwa beim gemeinsamen Tanzen) ${ }^{23}$ durch gut sicht- und hörbare Rückversicherungs- und Steuerungsphänomene unterstützt. Das geschieht im vorliegenden Beispiel vor allem durch Blickkontakt: Blickkontakt vor dem Auf-

23 Gemeinsames Tanzen ist ein Prototyp für die professionelle Steigerung von Konfigurationsherstellungen und -auflösungen, die zu hoch unwahrscheinlichen Ko-Ordinierungsleistungen führt (vgl. dazu Loenhoff 2003 und Müller/Bohle 2007 mit vielen weiteren Literaturhinweisen zur Phänomenologie des Tanzens). 
stehen, Blick in die Runde, rhythmische Aufwärtsbewegung des Kopfes als Einladung zum Weitergehen und Rückversicherung der Bereitschaft zur Auflösung der Konfiguration:

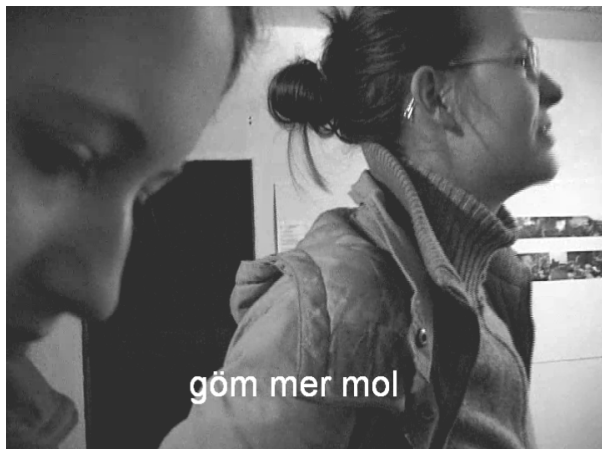

Z. 82: Demonstrative Blickorientierung Claudias zu einem anderen („nächsten“) Wahrnehmungsobjekt

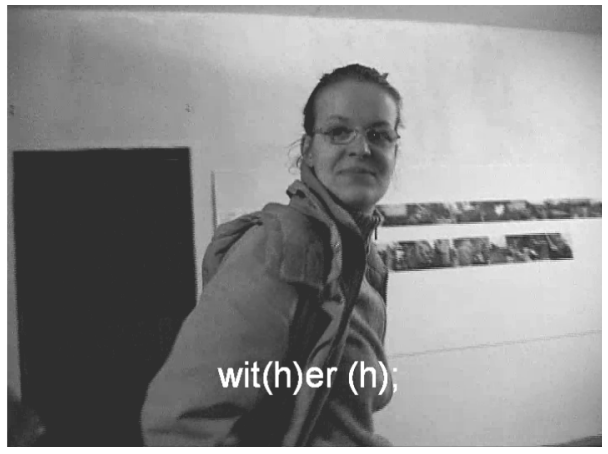

Z. 83: Rückversicherung vor dem Weggehen: Claudia blickt zu Melanie (nicht im Bild)

Nicht zufällig erfolgt in dieser für die Auflösung der Konfiguration kritischen Sequenz auch ein sprachlicher Steuerungshinweis (,göm mer mol witer"), der punktgenau das anstehende kommunikative Koordinationsproblem thematisiert: die Bewegung, auf die es ankommt (weiter gehen), die Gemeinsamkeit dieser Bewegung (wir) und die Modalisierung der Herbeiführung des Aufbruchs im Sinne eines handlungsauslösenden Vorschlags (mal).

Es versteht sich von selbst, dass diese Beschreibung analytisch noch zu verfeinern und auszubauen wäre. Man sieht aber vielleicht auch so, wie sich aus dem „hier“ als Bewegungsraum zwangsläufig eine Daueraufgabe der Ko-Ordinierung ergibt, um der Gefahr der Selbstauflösung der Interaktion durch Bewegung zu entgehen. ${ }^{24}$

24 „Koordinierung“ bzw. „Koordination“ ist ein in Interaktionsstudien immer wieder anzutreffender Terminus. Er wird z.B. auch schon bei Kendon in vergleichbaren Zusammenhängen gebraucht. Explizit eingeführt wird er bei Deppermann/Schmitt (2007). 
Ko-Orientierung und Ko-Ordinierung sind grundlegende Anforderungen, die illustrieren, was Situierung konkret bedeutet und wie sie die Sensorik und Motorik der InteraktionsteilnehmerInnen als Ressource von Anwesenheit nutzt. Situierung erschöpft sich aber nicht darin. Die durch Ko-Orientierung und Ko-Ordinierung interaktiv relevant gemachten Wahrnehmungen und Bewegungen sind immer schon Teil einer sozialen Handlungspraxis.

\subsection{Handlung}

Wenn man die soziale Praxis des gemeinsamen Ausstellungsbesuchs erfassen möchte, kann man sich nicht (ethologisch) auf Wahrnehmung und Bewegung beschränken. Ko-Orientierungs- und Ko-Ordinierungsaktivitäten müssen auf das bezogen werden, was im Raum an sozialen Handlungen möglich und wahrscheinlich ist. In dieser Hinsicht ist das „hier“ auch ein Handlungsraum. Ko-Orientierungen und Ko-Ordinierungen sind ja kein Selbstzweck, sondern eingebettet in eine voraussetzungsreiche soziale Praxis, die Wahrnehmungen und Bewegungen sozial sinnvoll macht und gleichzeitig eine soziale Raumkognition auf Seiten der Teilnehmenden voraussetzt.

Wenn wir uns für diese soziale Praxis interessieren, können wir nicht länger von dem abstrahieren, was gesprochen und gehört wird. Die soziale Praxis des gemeinsamen Ausstellungsbesuchs ist nicht nur eine Praxis wechselseitig abgestimmter Wahrnehmung und Bewegung, sondern eben auch eine Praxis des Redens über Kunst, eine gesellschaftliche Praxis der Kunstkommunikation.

Kunstkommunikation impliziert, das zeigt dieses Beispiel sehr anschaulich, eine Reihe typischer Zugzwänge, zu denen das Beschreiben (Was gibt es zu sehen?), das Deuten (Was steckt dahinter?), das Erläutern (Was weiß man darüber?) und das Bewerten (Was ist davon zu halten?) gehören. Es handelt sich jeweils um kleinformatige interaktive Aufgaben mit typischen sprachlichen Mitteln und Formen. Typisch für das Beschreiben ist z.B. die Verwendung der verba sentiendi, um z.B. Gesehenes im Sinne eines Anscheins zu thematisieren: das gseht aber us wie so ne so ne plastilinknät. Z. 1516; typisch für das Deuten ist z.B. die Thematisierung einer Mitteilungsabsicht im Modus des unsicheren Für-Wahr-Haltens (Polenz 1988, S. 214): ja sött sött das d dsch schwiiz darstelle: Z. 18-19; typisch für das Erläutern ist z.B. die Thematisierung von (Nicht)Wissen: also ich weiss nid weles land die form het: Z. 37-39; typisch für das Bewerten ist der Ausdruck von Ge- oder Missfallen: hö das find i no cool toll (im transkribierten Ausschnitt nicht enthalten). ${ }^{25}$

Auch ohne an dieser Stelle in eine vertiefende Analyse einzusteigen, sieht man leicht, dass mit diesen Aufgaben der Kunstkommunikation spe-

$25 \quad$ Vgl. dazu Hausendorf (2006). 
ziell die Themenorganisation als Aufgabenbereich in den Vordergrund rückt, also die Organisation von Beiträgen zu Themen (siehe oben 2.). Es hat den Anschein, dass das Finden einer Konfiguration (hier: das Verweilen vor dem Kunstwerk) das Zurücktreten der Situierung hinter stärker sprechund zuhörorientierte Interaktionsaufgaben nach sich zieht. Interessant ist schließlich auch, dass und wie die Kunstkommunikation im Angesicht des Kunstwerks gleichwohl auf Ko-Orientierung und Ko-Ordination angewiesen ist, genauer gesagt: auf die Konstitution dessen, was in den oben angedeuteten Fragen des Deutens, Beschreibens, Erläuterns und Bewertens immer schon impliziert ist: das Kunstwerk als gesichertes Wahrnehmungsobjekt ${ }^{26}$ Vielleicht kann man behaupten, dass gerade in der Konfiguration des gemeinsamen Verweilens und der in dieser Konfiguration sinnfällig werdenden Wahrnehmungsmuße (Oevermann 1996) die Zugzwänge des Beschreibens, Deutens, Erläuterns und Bewertens aufkommen, die für die Kunstkommunikation so charakteristisch sind. Ausstellungsmacher und Ausstellungsmacherinnen könnten dann auf die Idee kommen, dass man Interaktionsteilnehmer nur dazu bringen muss, gemeinsam in Wahrnehmungsmuße vor einem „Objekt“" zu verweilen, damit Kunstkommunikation als soziale Praxis wahrscheinlich wird. ${ }^{27}$

Wenn der Raum, zusammenfassend gesagt, ein Handlungsraum ist, dann ist neben der Ko-Orientierung und Ko-Ordinierung auch die KoOperation der Beteiligten eine interaktive Anforderung: Ko-Operation im Hinblick auf die für die fragliche soziale Praxis relevanten kommunikativen Zugzwänge und Aufgaben. Selbstverständlich ist eine solche Ko-Operation nicht. Wenn man an Ausstellungsbesuche mit Kindern denkt, weiß man, wie anspruchsvoll diese Anforderung der Ko-Operation sein kann - und was sie, nebenbei bemerkt, an Sozial- und Körperdisziplinierung immer schon voraussetzt.

Im Falle der Ko-Orientierung und der Ko-Ordinierung liegt der Raumbezug der Interaktion auf der Hand. Man könnte sich aber fragen, ob dagegen die Ko-Operation nicht mehr oder weniger unabhängig vom Raum vonstatten geht. Dass dies nicht der Fall ist, wird sofort deutlich, wenn wir uns den Raum, in dem die fragliche Episode spielt, genauer anschauen.

\subsection{Spielen}

Der Raum, der die Anwesenden umgibt, ist keine tabula rasa. Die Situierung, wie sie bislang mit Bezug auf Wahrnehmung, Bewegung und Handlung beschrieben worden ist, schließt vielmehr in all ihren Facetten an raumbasierte

\footnotetext{
$26 \quad$ Speziell dazu Vom Lehn/Heath (2007)

27 Das funktioniert vor allem dann, wenn es Anknüpfungspunkte im Raum gibt, die einen Kunstverdacht unterstützen. Im vorliegenden Beispiel ist das zweifellos der Fall - darauf kommen wir gleich noch zu sprechen (siehe unten 5.4).
} 
Kommunikation an. ${ }^{28}$ Man kann das so verstehen, dass das „hier“ immer auch ein Spielraum ist, eine Bühne, die betreten und auf der aufgetreten werden will. Mit dieser Begriffswahl sollen keine spieltheoretischen oder anspruchsvollen theatermetaphorischen Ambitionen verknüpft werden. Es geht darum, dass der Raum, der die Anwesenden umgibt, mit dem Naheliegenden lockt, und zwar nicht erst mit Bezug auf die in ihm möglichen kooperativen Handlungen, sondern schon mit Bezug auf die in ihm möglichen Ko-Orientierungen und Ko-Ordinierungen. Die Situierung kommt so gesehen zwangsläufig einer Aktivierung räumlicher Anschlussmöglichkeiten gleich.

Wenn man anfängt, den Raum so zu sehen, ist er voll von wahrnehmungs-, bewegungs- und handlungsrelevanten Markierungen, die wir in der Regel ohne langes Nachdenken durch koorientierte Wahrnehmung, koordinierte Bewegung und kooperatives Handeln ,aktivieren“. Bedeutung entsteht dabei unmittelbar durch Bewegung, auf Schritt und Tritt, wenn man so will.

Man übersieht schnell, dass es ja alles andere als selbstverständlich ist, was in einem Raum unsere Wahrnehmung anzieht und Bewegungen provoziert. Im Raum selbst gibt es dafür eine Art unsichtbarer Markierungen, denen wir in vielen Fällen folgen: Attraktionen für Wahrnehmung, Zonen von Betretbarkeit und Zugänglichkeit, Fokuszonen zum Verweilen, Tabuzonen zum Auslassen. Es gibt Räume, in denen die Markierung von relevanten Wahrnehmungs-, Bewegungs- und Handlungsräumen sofort sehr anschaulich wird. Dazu gehören Mehrzwecksporthallen: ${ }^{29}$
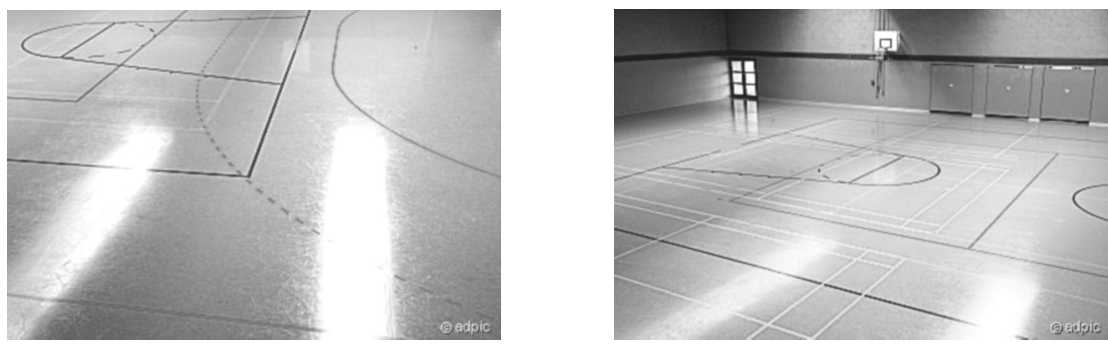

Abb. 3 u. 4: Markierungen im Raum ${ }^{30}$

28 Begriff und Konzept von raumbasierter Kommunikation werden in Kesselheim/Hausendorf (2007) eingeführt. Im Folgenden wird dieses Konzept mit Bezug auf die Konstitution des Spielraums entwickelt.

29 Wir kommen auf das bislang behandelte Beispiel des Ausstellungsraumes noch zurück, wollen den Gedankengang im Folgenden aber zunächst an einem besonders klaren Fall veranschaulichen.

30 Quelle: www.adpic.de/data/picture/detail/turnhalle_68745.jpg; www.adpic.de/data/picture/detail/turnhalle_68744.jpg (Stand Oktober 2009). 
Die farblich unterschiedlich markierten Linien und Striche auf dem Hallenboden illustrieren in großer Anschaulichkeit zwei Beobachtungen:

- Die Markierungen sind Vorgaben, die der Raum für mögliche Spiele macht, Anschlussmöglichkeiten für Anwesende, an die Interaktion anschließen kann, aber nicht anschließen muss: Man kann in einer Turnhalle alles Mögliche machen, auch ein sit in mit Kaffee und Kuchen. Ihren speziellen Mehrwert für die Interaktion kann die Turnhalle aber erst entfalten, wenn ihre Linien und Striche als Markierungen von Spielräumen genutzt werden.

- Die Markierungen müssen also verstanden werden in dem Sinne, dass Anwesende auf die Idee kommen, einen Unterschied zu machen zwischen der Information, dass da etwas farblich markiert ist auf dem Hallenboden (Linien), und der Mitteilung, dass hinter diesen Markierungen (Linien) Spieloptionen stecken.

Es ist die Auffälligkeit (graphisch und farblich), die ein solches Verstehen in diesen Fällen wohl fast unvermeidbar macht (auch wenn man nicht weiß, welche Spiele „hier“ wie zu spielen sind), die Unwahrscheinlichkeit solcher Linien, die Kommunikation in solchen Fällen anlaufen lässt - anders als im Falle der Kratzer auf dem Fußboden, die wir ebenfalls sehen mögen, aber hinter denen wir in der Regel keine Mitteilungsabsicht verstehen. ${ }^{31}$

Was es heißt, den Raum auf diese Weise zu „verstehen“, zeigt die Nutzung der Mehrzwecksporthalle in großer Anschaulichkeit:
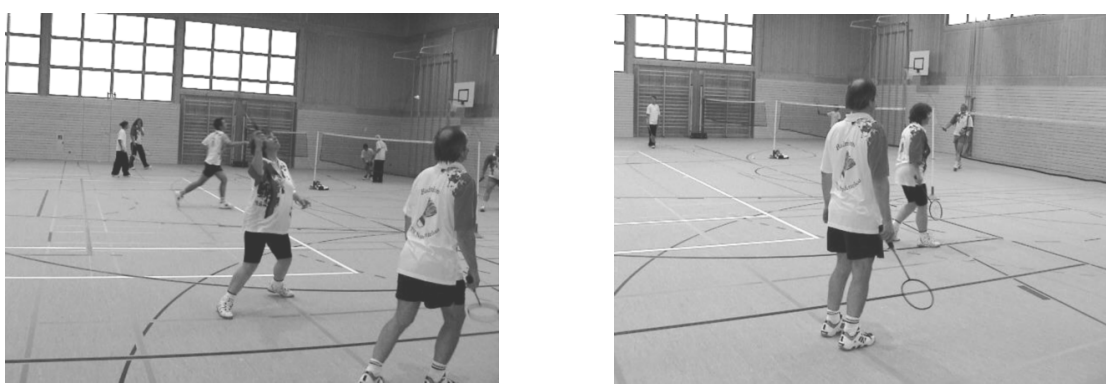

Abb. 5 u. 6: den Raum verstehen ${ }^{32}$

Es handelt sich um ein in Ko-Orientierung, Ko-Ordinierung und KoOperation ausgedrücktes Verstehen des Raumes, das sich sogar auf diesen Zufallsbildchen ausdrückt, wenn man z.B. darauf schaut, wie der „Spie-

$31 \quad$ Man kann das kommunikationstheoretisch (z.B. mit Luhmann 1984), aber auch zeichentheoretisch (z.B. mit Keller 1995) begründen.

32 Quelle: www.naunet.de/Landshuter/turnhalle/DSC00072.jpg; www.naunet.de/Landshuter/turnhalle/DS C00071. jpg (Stand: Oktober 2009). 
ler" im Vordergrund die blaue Linie durch seine Position in ihrer interaktiven Relevanz bestätigt - und andere gleichzeitig interaktiv außer Kraft setzt. Ist es nicht sehr illustrativ zu sehen, wie der Raum durch solche Markierungen zu einer Bühne für sehr voraussetzungsreiche Interaktionsspiele gemacht werden kann? Wer solche Interaktionsspiele beobachten will, wird gut daran tun, sich die Markierungen im Raum sehr genau anzugucken.

Auch wenn es in den meisten Räumen, in denen wir unsere Alltagsspiel(ch)e(n) machen, keine farblichen Markierungen und erst recht keinen „Linienrichter“" gibt, so gibt es doch fast immer eine Lesbarkeit des Raumes im Hinblick auf seine wahrnehmungs-, bewegungs- und handlungsrelevanten Markierungen. Das „hier“ ist also auch ein Spielraum und die Ko-Aktivierung dieses Raumes eine weitere interaktive Anforderung. Wir könn(t)en jedenfalls viel über unsere Alltagsräume lernen, wenn wir sie in dieser Weise als Spielfelder zu beobachten anfangen und nach ihren Markierungen fragen. Eine solche interaktionstheoretisch fundierte und linguistisch informierte Analyse des Raumes und seiner Markierungen steht noch am Anfang. Auch die Interaktionsforschung in der Tradition der "Context Analysis“ (siehe oben 3.) hat davor bis in unsere Tage hinein zurückgescheut. ${ }^{33} \mathrm{An}$ knüpfen ließe sich aber beispielsweise an semiotische Analysen zur zeichenbasierten Orientierung im Raum. ${ }^{34}$

Um auf die hier untersuchte soziale Praxis der Kunstkommunikation in der Ausstellung zurückzukommen: Worauf unsere Teilnehmer ihre Wahrnehmung ko-orientieren und wie sie ihre Bewegungen ko-ordinieren, ist ja durch die Gestaltung des Ausstellungsraumes bereits hochgradig nahegelegt: Es handelt sich um einen unmöblierten Raum, an dessen Wänden z.T. Objekte angebracht sind und in dessen Mitte auf dem Fußboden das oben im Mittelpunkt von Wahrnehmung, Bewegung und Handlung stehende Objekt platziert ist.

33 Vgl. aber etwa am Beispiel des Klassenraums Willems/Eichholz (2008); Breidenstein (2004); am Beispiel der Orientierung in Bibliotheken Crabtree (2000) oder am Beispiel des Museums Kesselheim/Hausendorf (2007). In evolutionärer Hinsicht sehr instruktiv sind die Ausführungen zum „humanisierten Raum“ bei Leroi-Gourhan (1980, S. 395 ff.).

34 Einschlägig dafür z.B. die Analysen zur Raumorientierung im Straßenverkehr in Posner/ Krampen (Hg.) (1995). Vgl. auch Schmauks (2002) am Beispiel der Raumorientierung beim Wandern. Hinweise zur semiotischen Analyse der Museumskommunikation finden sich in Kesselheim/Hausendorf (2007). Siehe dazu auch den Beitrag von Habscheid et al. (in diesem Band) zu Markierungen und ihrer Interpretation in Notfalleinsätzen. 


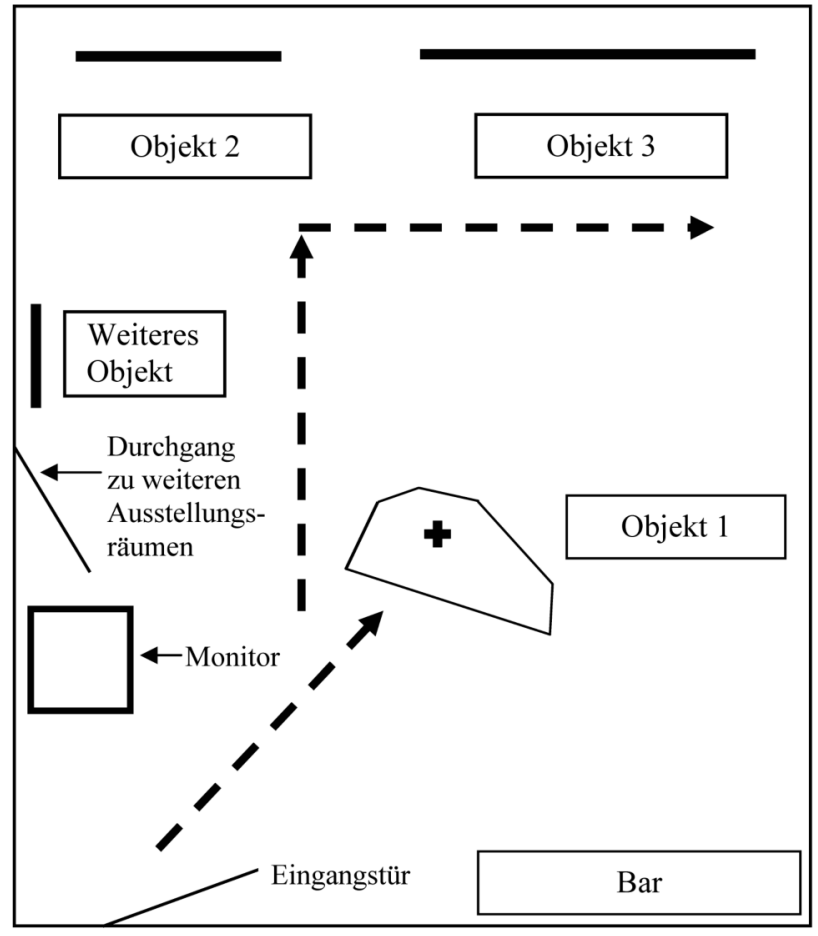

Legende:

$\longrightarrow-\rightarrow=$ grober Laufweg von $\mathrm{C}, \mathrm{M}$ und $\mathrm{N}$

$=$ an der Wand hängende Objekte

Abb. 7: Skizze des Ausstellungsraumes ${ }^{35}$

Scheinbar wie von selbst wirken die auf diese Weise als „Exponate“ auffällig gemachten Objekte als Attraktoren für Wahrnehmung, Bewegung und Handlung. An dieser Stelle kommt über den Raum und seine Gestaltung eine ganze Kultur der Ausstellungskommunikation als raumgebundene kommunikative Ressource ins Spiel. Indem das, was wir oben im Einzelnen an Ko-Orientierung, -Ordination und -Operation beschrieben haben, den Exponat-Charakter der Objekte zur Geltung bringt, haben wir es also längst mit Ko-Aktivierung zu tun - auch wenn das in diesem Fall nicht so anschaulich sein mag wie beim Spiel in der Mehrzwecksporthalle, das scheinbar wie von selbst der Markierung von Spielräumen folgt. Auch die fokale Zone vor einem Objekt und um ein Objekt herum ist insofern im wahrsten Sinne des Wortes ein Spielraum.

35 Diese Skizze stammt im Original von Sabina Gröner, der ich auch die Aufzeichnung und Transkription verdanke (siehe oben Anm. 17). 
Wir haben es in unserem Fall des Ausstellungsbesuches - im Grunde nicht anders als im Fall des Spielens in der Mehrzwecksporthalle - damit zu tun, dass die beschriebenen Ko-Orientierungen, -ordinationen und -operationen systematisch durch die Gestaltung des Raums nahegelegt sind: in diesem Fall (anders als in der Mehrzwecksporthalle) durch eine ganze Tradition der Ausstellung von Objekten in einem ansonsten so gut wie leeren weißen Raum, der vor allem durch seine Leere (durch größtmögliche Abwesenheit von Ablenkungen) signifikant ist und damit alles unter Kunstverdacht stellt, was in einem solchen Raum nicht offenkundig funktional motiviert ist (wie z.B. eine Steckdose oder die Beschläge an Türen und Fenstern):

Die ideale Galerie hält vom Kunstwerk alle Hinweise fern, welche die Tatsache, daß es „Kunst“ ist, stören könnten. Sie schirmt das Werk von allem ab, was seiner Selbstbestimmung hinderlich in den Weg tritt. Dies verleiht dem Raum eine gesteigerte Präsenz [...] Etwas von der Heiligkeit der Kirche, etwas von der Gemessenheit des Gerichtssaals, etwas vom Geheimnis des Forschungslabors verbindet sich mit chicem Design zu einem einzigartigen Kultraum der Ästhetik. ... Eine Galerie wird nach Gesetzen errichtet, die so streng sind wie diejenigen, die für eine mittelalterliche Kirche galten. Die äußere Welt darf nicht hineingelassen werden [...] Die Wände sind weiß getüncht. Die Decke wird zur Lichtquelle. [...] In dieser Umgebung wird ein Standaschenbecher fast zu einem sakralen Gegenstand, ebenso wie der Feuerlöscher in einem modernen Museum einfach nicht mehr wie ein Feuerlöscher aussieht, sondern wie ein ästhetisches Scherzrätsel. Hier erreicht die Moderne die endgültige Umwandlung der Alltagswahrnehmung zu einer Wahrnehmung rein formaler Werte. (O'Doherty 1996, S. 9 ff.)

Dass wir an diesen white cube so sehr gewöhnt sind, ändert, wie Brian O'Doherty in seinem viel zitierten Essay gezeigt hat, nichts an der Unwahrscheinlichkeit dieser Art von Raum gewordener Ausstellungskommunikation. Es ist also schon sehr viel passiert, bevor wir den Raum betreten. Ausstellungsmacher können davon ein Lied singen. ${ }^{36}$

Wenn wir uns für Situierung interessieren, sollten wir uns die raumgebundene und raumbasierte Kommunikation nicht entgehen lassen. Wir verstehen sonst die Situierung nicht.

\section{Fazit}

Die anhand der Fallbeobachtungen illustrierten Anforderungen verdeutlichen, wie das „hier“, der Raum der Interaktion, durch Situierungsaktivitäten ins Spiel kommt. Die folgende Abbildung fasst diese Anforderungen im Sinne charakteristischer Situierungsprobleme zusammen:

36 Sehr instruktiv unter dem Aspekt der Veränderung von Wahrnehmungskulturen durch Ausstellungstechniken sind die „Zurichtung des Umraums“ in Ausstellungen seit dem 17. Jahrhundert und ihre jeweils zeitgenössische Reflexion (Hinweise dazu bei Schwarte 2005, S. 292 ff. und natürlich auch bei O'Doherty 1996). 


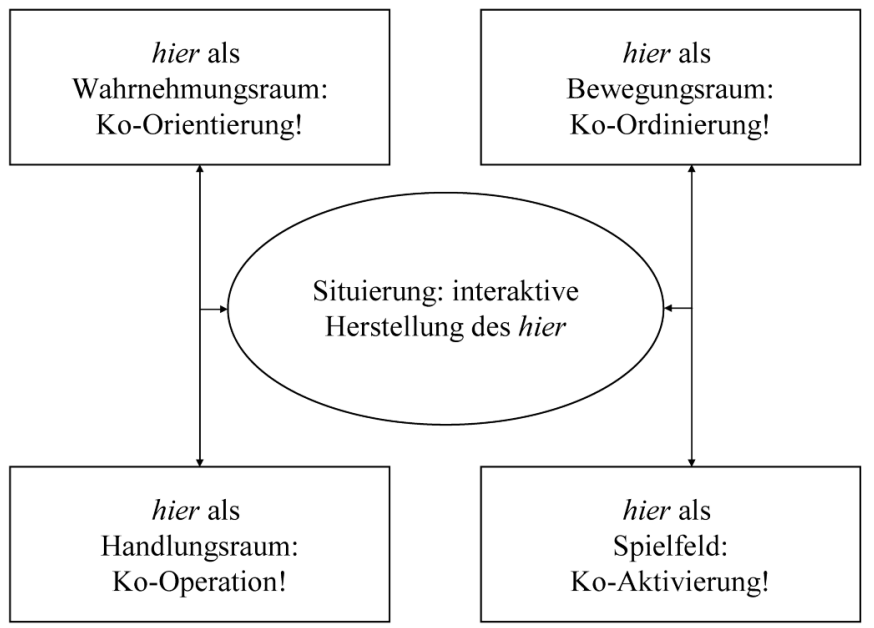

Abb. 8: Situierungsprobleme

Das „hier“ ist zunächst in einem elementaren Sinne ein Raum für Wahrnehmungen, in dem sich die Beteiligten zurechtfinden und orientieren müssen und in dem sie sich ihre Orientierungen wechselseitig aufzeigen müssen. Das Problem, das daraus für die Interaktion erwächst, ist ein Ko-Orientierungsproblem. Mit der Bearbeitung und Lösung dieses Problems wird der Raum für die Interaktion sensorisch erschlossen und in seiner Wahrnehmbarkeit relevant gemacht. Die interaktive Herstellung der räumlichen Bedingungen der Sprechsituation geschieht maßgeblich in diesem Medium der Ko-Orientierung und den sich dabei vollziehenden Wahrnehmungswahrnehmungen.

Das „hier“ ist des Weiteren ein Raum für Bewegungen, in dem sich die Beteiligten positionieren müssen und in dem sie sich ihre Positionen und Navigationen wechselseitig aufzeigen müssen. Das Problem, das daraus für die Interaktion erwächst, ist ein Ko-Ordinierungsproblem. Mit der Bearbeitung und Lösung dieses Problems wird der Raum für die Interaktion motorisch erschlossen und in seiner Betret- und Begehbarkeit interaktiv relevant gemacht. Das Ergebnis der Ko-Ordinierung sind typische Konfigurationen der Körper der Anwesenden im Raum, ohne die keine Interaktion denkbar wäre: Begriffe wie „face-to-face“, „face-to-back“ oder „side-by-side“ deuten solche Konfigurationen an, lenken aber bereits von der Vielfalt der unter Anwesenden möglichen Positionierungs- und Navigationsmöglichkeiten ab. ${ }^{37}$

Das „hier“ ist weiterhin ein Raum für Handlungen, in dem die Beteiligten nicht nur hin- und herschauen und hin- und herlaufen, sondern agieren und in dem sie sich ihr Agieren wechselseitig aufzeigen müssen, indem sie ihre Wahrnehmungen und Bewegungen als sozial sinnvoll demonstrieren

37 Weiterführend sind hier die von A. Kendon eingeführten „focus formation systems“ (Kendon 1990). 
und aufeinander abstimmen. Das Problem, das daraus für die Interaktion erwächst, ist ein Ko-Operationsproblem. Mit der Bearbeitung und Lösung dieses Problems wird der Raum für die Interaktion handlungspraktisch erschlossen und in seinem Handlungspotential (in seiner Behandelbarkeit) interaktiv relevant gemacht. Das Ergebnis der Ko-Operation ist dann eine bestimmte soziale Praxis, die den Wahrnehmungs- und Bewegungsraum mehr oder weniger intensiv in Anspruch nehmen kann: Ein Gespräch am runden Tisch, das den Körper etwa zur Hälfte unsichtbar lässt und - solange alle sitzen bleiben - allenfalls das motorische Potential der „Oberkörper“, des Kopfes und der Arme ausschöpfen mag (siehe dazu das bei Schmitt/ Deppermann in diesem Band analysierte Beispiel), ist im Hinblick auf KoOrientierungen und Ko-Ordinierungen der Anwesenden, also sensorisch und motorisch, viel weniger anspruchsvoll als ein Ballspiel ein Boxkampf oder ein Tan:

Je intensiver der Wahrnehmungs- und Bewegungsraum durch die Interaktion in Anspruch genommen wird, desto anschaulicher tritt schließlich hervor, dass das „hier“ auch ein Raum für (Sprach- und Interaktions)Spiele, ein Spielfeld, ist, das die Beteiligten durch ihre Spielzüge („,moves“i.S.v. Goffman 1974) aktivieren müssen. Das Problem, das daraus für die Interaktion erwächst, ist ein Ko-Aktivierungsproblem. Mit der Bearbeitung und Lösung dieses Problems wird der Raum für die Interaktion gewissermaßen theatral erschlossen und in seinem Potential als Bühne für Interaktions- und Sprachspiele interaktiv relevant gemacht. „Interaktion im Raum“ heißt auch, dass Ko-Orientierung, Ko-Ordinierung und Ko-Operation nicht immer wieder voraussetzungslos wie von Neuem zustande gebracht werden müssen, sondern mittels gesellschaftlicher Spielräume (Bühnen) an etablierte Interaktionsrituale anschließen können. Der Raum der Interaktion ist in diesem Sinne fast immer auch ein gesellschaftlicher Spielraum.

Insbesondere dort, wo das Zusammensein institutionalisiert und organisiert ist, kann man auf hoch voraussetzungsreiche Spielräume mit wahrnehmungs-, bewegungs- und handlungsrelevanten Markierungen treffen, die ihren Ausdruck in der Architektur von Gebäuden (wie dem Gericht, dem Krankenhaus oder der Universität), der Ausgestaltung ihrer Innenräume bis hin zur Möblierung des Raums gefunden haben. Es sind solche Spielräume der Gesellschaft, in denen sich, interaktionstheoretisch formuliert, der Anschluss von Interaktion an Gesellschaft alltäglich vollzieht, indem sich Situierung und Kontextualisierung (siehe oben 2.) auf eine sehr effektive Weise überlappen. Hier vor allem muss man suchen, wenn man den viel zitierten Mikro-Makro-Link (Habscheid 2000) zwischen Interaktion und Gesellschaft finden möchte.

Wenn man so argumentiert, erweist sich das Verhältnis zwischen KoOrientierung, Ko-Ordinierung, Ko-Operation und Ko-Aktivierung als ein Verhältnis zunehmender Voraussetzungshaftigkeit: gewissermaßen am An- 
fang steht die Ko-Orientierung (damit beginnt ja die Interaktion: ein bezeichnender Zusammenfall von Interaktionseröffnung und Situierung), gewissermaßen am Ende steht die Ko-Aktivierung des Spielraumes, die alle anderen Situierungs-Anforderungen einschließt.

Mit ihrer durchgängigen Betonung von Wahrnehmung, Bewegung, Handlung und Spiel könnten die vorgestellten Überlegungen womöglich als ein Beitrag zur Analyse der Welt des Sports verstanden werden, in der es auf Sprache und die Beobachtung von Sprache (durch Linguisten und Linguistinnen) nicht ankommt. Das wäre ein Missverständnis. Es kommt mir nur so vor, als ob die Welt des Sports - stärker vielleicht noch als die in dieser Hinsicht oft in Anspruch genommene Welt des Theaters ${ }^{38}$ - besonders geeignet wäre, zu verstehen, was Situierung bedeutet und welche Rolle die Sprache dabei spielt.

\section{Literatur}

Auer, Peter (2004): Sprache, Grenze, Raum. In: Zeitschrift für Sprachwissenschaft 23, S. 149-180.

Peter Auer (i.d.Bd.): Sprachliche Landschaften. Die Formung des Raums durch die geschriebene Sprache

Backhaus, Peter (2007): Linguistic landscapes. A comparative study of urban multilingualism in Tokyo. Clevedon.

Bernhard, Gerald/Siller-Runggaldier, Heidi (2008): Sprache im Raum - Raum in der Sprache. Akten der sprachwissenschaftlichen Sektion des Deutschen Italianistentages in Bochum, 23.-25. März 2006. (= Spazi comunicativi 4). Frankfurt a.M.

Bickel, Balthasar/Nichols, Johanna (2006): Oceania, the Pacific Rim and the theory of linguistic areas. Proceedings of the 32nd Annual Meeting of the Berkeley Linguistics Society. Internet: www.uni-leipzig.de/ bickel/research/papers/bickel_nichols_bls32.pdf (Stand: Oktober 2009).

Bloom, Paul Peterson (Hg.) (1996): Language and space. Cambridge, MA.

Breidenstein, Georg (2004): KlassenRäume - eine Analyse räumlicher Bedingungen und Effekte des Schülerhandelns. In: Zeitschrift für qualitative Bildungs-, Beratungs- und Sozialforschung 5, 1, S. 87-107.

Bühler, Karl (1982): Sprachtheorie. Die Darstellungsfunktion der Sprache. Stuttgart/ New York.

Buss, Mareike/Habscheid, Stephan/Jautz, Sabine (Hg.) (2009): Theatralität des sprachlichen Handelns. Eine Metaphorik zwischen Linguistik und Kuturwissenschaften. München.

Crabtree, Andy (2000): Remarks on the social organisation of space and place. In: Journal of Mundane Behavior 1/1, S. 25-44.

38 Vgl. dazu den gerade erschienenen Sammelband Buss/Habscheid/Jautz (Hg.) (2009). 
Deppermann, Arnulf/Schmitt, Reinhold (2007): Koordination. Zur Begründung eines neuen Forschungsgegenstandes. In: Schmitt (Hg.), S. 15-54.

Döring, Jörg/Thielmann, Tristan (Hg.) (2008): Spatial turn. Das Raumparadigma in den Kultur- und Sozialwissenschaften. Bielefeld.

Dünne, Jörg/Günzel, Stephan (Hg.) (2006): Raumtheorie. Grundlagentexte aus Philosophie und Kulturwissenschaften. Frankfurt a.M.

Frank, Roslyn M./Dirven, René/Ziemke, Tom/Bernárdez, Enrique (Hg.) (2008): Body, language and mind. Bd. 2: Sociocultural situatedness. (= Cognitive Linguistics Research 35.2). Berlin/New York.

Fricke, Ellen (2007): Origo, Geste und Raum - Lokaldeixis im Deutschen. (= Linguistik - Impulse \& Tendenzen 24). Berlin/New York.

Garfinkel, Harold (1967): Studies in ethnomethodology. Englewood Cliffs.

Goffman, Erving (1964): The neglected situation. In: Gumperz, John J./Hymes Dell (Hg.): The ethnography of communication. In: American Anthroplogist, 6, 2, S. 133-136.

Goffman, Erving (1974): Das Individuum im öffentlichen Austausch. Mikrostudien zur öffentlichen Ordnung. Frankfurt a.M.

Goffman, Erving (1977): Rahmen-Analyse. Ein Versuch über die Organisation von Alltagserfahrungen. Frankfurt a.M.

Goodwin, Charles (2000): Action and embodiment within situated human interaction. In: Journal of Pragmatics 32, S. 1489-1522.

Gülich, Elisabeth/Mondada, Lorenza (2008): Konversationsanalyse. Eine Einführung am Beispiel des Französischen. (= Romanistische Arbeitshefte 52). Tübingen.

Habscheid, Stephan (2000): Das „Mikro-Makro-Problem“ in der Gesprächsforschung. In: Gesprächsforschung - Online-Zeitschrift zur verbalen Interaktion 1, S. 125 148. Internet: www.gespraechsforschung-ozs.de/heft2000/ga-habscheid.pdf (Stand: Oktober 2009).

Habscheid, Stephan et al. (i.d.Bd.): Artikulationsarbeit und mediengestützte Ortserkundung. Multimodale und multilokale Kommunikation in Notfalleinsätzen

Hanks, William F. (1990): Referential practice. Language and lived space among the Maya. Chicago.

Hausendorf, Heiko (1992): Gespräch als System. Linguistische Aspekte einer Soziologie der Interaktion. Opladen.

Hausendorf, Heiko (2000): Zugehörigkeit durch Sprache. Eine linguistische Studie am Beispiel der deutschen Wiedervereinigung. (= Reihe Germanistische Linguistik 215). Tübingen.

Hausendorf, Heiko (2003): Deixis and speech situation revisited. The mechanism of perceived perception. In: Lenz, Friedrich (Hg.): Deictic conceptualisiation of space, time and person. (= Pragmatics \& beyond, N.S. 112). Amsterdam, S. 249-269.

Hausendorf, Heiko (2006): Gibt es eine Sprache der Kunstkommunikation? Linguistische Zugangsweisen zu einer interdisziplinären Thematik. In: Paragrana. Internationale Zeitschrift für Historische Anthropologie 15, S. 65-98. 
Hausendorf, Heiko (2007): Was kommt als Nächstes? Fokussierungen revisited. In: Hausendorf (Hg.), S. 221-246.

Hausendorf, Heiko (Hg.) (2007): Gespräch als Prozess. Linguistische Aspekte der Zeitlichkeit verbaler Interaktion. (= Studien zur Deutschen Sprache 37). Tübingen.

Hausendorf, Heiko/Kesselheim, Wolfgang (2008): Textlinguistik fürs Examen. (= Linguistik fürs Examen 5). Göttingen.

Heath, Christian/Hindmarsh, Jon (2000): Configuring action in objects. From mutual space to media space. In: Mind, Culture, and Activity 7, S. 81-104.

Herrmann, Theo/Schweizer, Karin (1998): Sprechen über Raum. Sprachliches Lokalisieren und seine kognitiven Grundlagen. Bern.

Hickmann, Maya/Robert, Stéphane (2006): Introduction. Space, language, and cognition. Some new challenges. In: Hickmann/Robert (Hg.), S. 1-15.

Hickmann, Maya/Robert, Stéphane (Hg.) (2006): Space in languages. Linguistic systems and cognitive categories. (= Typological Studies in Language 66). Amsterdam.

Hindmarsh, Jon/Heath, Christian (2000): Embodied reference. A study of deixis in workplace interaction. In: Journal of Pragmatics 32, S. 1855-1878.

Jones, Christopher B./Purves, Ross S./Clough, Paul D./Joho, Hideo (2008): Modelling vague places with knowledge from the Web. In: International Journal of Geographic Information Systems, 22, 10, S. 1045-1065.

Kallmeyer, Werner/Schütze, Fritz (1976): Konversationsanalyse. In: Studium Linguistik 1, S. 1-28.

Keller, Rudi (1995): Zeichentheorie. Zu einer Theorie semiotischen Wissens. Tübingen/Basel.

Kendon, Adam (1990): Conducting interaction. Patterns of behavior in focused encounters. (Studies in Interactional Sociolinguistics 7). Cambridge.

Kesselheim, Wolfgang (2009): Wissensvermittlung in der Museumsausstellung als Herausforderung für die Linguistik. In: Stenschke, Oliver (Hg.): Wissenstransfer und Diskurs. (= Transferwissenschaften 6). Frankfurt a.M. u.a., S. 245-266.

Kesselheim, Wolfgang/Hausendorf, Heiko (2007): Die Multimodalität der Ausstellungskommunikation. In: Schmitt (Hg.), S. 339-375.

Knoblauch, Hubert (2005): Video-Interaktions-Sequenzanalyse. In: Wulf/Zirfas (Hg.), S. 263-275.

Leroi-Gourhan, André (1980): Hand und Wort. Die Evolution von Technik, Sprache und Kunst. Frankfurt a.M.

Levinson, Stephen C. (1996): Language and space. In: Annual Review of Anthropology 25, S. 353-382.

Levinson, Stephen C. (2003): Space in language and cognition. Explorations in cognitive diversity. Cambridge.

Levinson, Stephen C./Wilkins, David (Hg.) (2006): Grammars of space. Explorations in cognitive diversity. Cambridge. 
Loenhoff, Jens (2002): Sensomotorische Bedingungen von Kommunikation und Handlung. In: Hahn, Kornelia/Meuser, Michael (Hg.): Körperrepräsentationen. Die Ordnung des Sozialen und der Körper. Konstanz, S. 45-69.

Loenhoff, Jens (2003): Grundlagen der kommunikativen Dimension von Körperbewegung und Tanz. In: Klinge, Antje/Leeker, Martina (Hg.): Tanz Kommunikation Praxis. Münster, S. 17-31.

Löw, Martina (2001): Raumsoziologie. Frankfurt a.M.

Luhmann, Niklas (1984): Soziale Systeme. Grundriß einer allgemeinen Theorie. Frankfurt a.M.

Mondada, Lorenza (2007): Interaktionsraum und Koordinierung. In: Schmitt (Hg.), S 55-93.

Mulder, Walter de (1996): Demonstratives as locating expressions. In: Pütz/Dirven (Hg.), S. 29-47.

Müller, Cornelia/Bohle, Ulrike (2007): Das Fundament fokussierter Interaktion. Zur Vorbereitung und Herstellung von Interaktionsräumen durch körperliche Koordination. In: Schmitt (Hg.), S. 129-165.

Norris, Sigrid (2004): Analyzing multimodal interaction. A methodological framework. New York.

O'Doherty, Brian (1996): In der weißen Zelle. Inside the white cube. (= Internationaler Merve-Diskurs 190). Berlin.

Oevermann, Ulrich (1996): Krise und Muße. Struktureigenschaften ästhetischer Erfahrung aus soziologischer Sicht. Internet: www.w-f-k.de/PDF-Dateien/Ulrich\%200ever mann,\%20Krise\%20und\%20Musse.pdf (Stand: Oktober 2009).

Park, Robert E. (1926): The urban community as a spatial pattern and a moral order. In: Burgess, Ernest W. (Hg.): The urban community. Selected papers from the Proceedings of the American Sociological Society. Chicago, S. 3-18.

Polenz, Peter von (1988): Deutsche Satzsemantik. Grundbegriffe des Zwischen-denZeilen-Lesens. Berlin/New York.

Posner, Roland/Krampen, Martin (Hg.) (1995): Kommunikation im Straßenverkehr. In: Zeitschrift für Semiotik 17, S. 1-2.

Pütz, Martin/Dirven, René (Hg.) (1996): The construal of space in language and thought. (= Cognitive Linguistics Research 8). Berlin.

Richardson, Tim/Jensen, Ole B. (2003): Linking discourse and space: Towards a cultural sociology of space in analysing spatial policy discourses. In: Urban Studies 40, S. $7-22$.

Schegloff, Emmanuel A./Sacks, Harvey (1973): Opening up closings. In: Semiotica 8, S. 289-327.

Schlögel, Karl (2003): Im Raume lesen wir die Zeit. Über Zivilisationsgeschichte und Geopolitik. München. 
Schlottmann, Antje (2005): Rekonstruktion alltäglicher Raumkonstruktionen. Eine Schnittstelle von Sozialgeographie und Geschichtswissenschaft? In: Geppert, Alexander C.T. (Hg.): Ortsgespräche. Raum und Kommunikation im 19. und 20. Jahrhundert. Bielefeld, S. 107-133.

Schmauks, Dagmar (2002): Orientierung im Raum. Zeichen für die Fortbewegung. (= Probleme der Semiotik 20). Tübingen.

Schmitt, Reinhold (2006): Videoaufzeichnungen als Grundlage für Interaktionsanalysen. In: Deutsche Sprache 34, S. 18-31.

Schmitt, Reinhold (Hg.) (2007): Koordination. Analysen zur multimodalen Interaktion. (= Studien zur Deutschen Sprache 38). Tübingen.

Schmitt, Reinhold/Deppermann, Arnulf (i.d.Bd.): Die multimodale Konstitution eines imaginären Raums als interaktive Problemlösung.

Schroer, Markus (2007): Räume, Orte, Grenzen. Auf dem Weg zu einer Soziologie des Raums. Frankfurt a.M.

Schroer, Markus (2008): „Bringing Space back in“-Zur Relevanz des Raums als soziologischer Kategorie. In: Döring/Thielmann, S. 125-148.

Schütz, Alfred (1972): Gemeinsam Musizieren. In: Schütz, Alfred: Gesammelte Aufsätze. Bd. II: Studien zur soziologischen Theorie. Den Haag, S. 129-150.

Schwarte, Ludger (2005): Das Einräumen von Bildlichkeit. Wahrnehmungshandlungen und Ausstellungsarchitektur. In: Wulf/Zirfas (Hg.), S. 279-299.

Schweizer, Harro (Hg.) (1985): Sprache und Raum. Ein Arbeitsbuch für das Lehren von Forschung. Stuttgart.

Scollon, Ron/Scollon, Suzie Wong (2003): Discourses in place. Language in the material world. London.

Vater, Heinz (1991): Einführung in die Raum-Linguistik. Hürth-Efferen.

Vom Lehn, Dirk/Heath, Christian (2007): Perspektiven der Kunst - Kunst der Perspektiven. In: Hausendorf, Heiko (Hg.): Vor dem Kunstwerk. Interdisziplinäre Aspekte des Sprechens und Schreibens über Kunst. München, S. 147-170.

Weigel, Sigrid (2002): Zum „topographical“ turn. Kartographie, Topographie und Raumkonzepte in den Kulturwissenschaften. In: KulturPoetik - Zeitschrift für kulturgeschichtliche Literaturwissenschaft 2, S. 151-165.

Weinrich, Harald (1993): Textgrammatik der deutschen Sprache. Mannheim/Leipzig/ Wien/Zürich.

Willems, Herbert/Eichholz, Daniela (2008): Die Räumlichkeit des Sozialen und die Sozialität des Raumes. Schule zum Beispiel. In: Willems, Herbert (Hg.): Lehr(er)buch Soziologie. Für die pädagogischen und soziologischen Studiengänge. Bd. 2. Wiesbaden, S. 865-907.

Wulf, Christoph/Zirfas, Jörg (Hg.) (2005): Ikonologie des Performativen. München. 
